\title{
Fault Diagnosis of Beam-Like Structure Using Modified Fuzzy Technique
}

\author{
Dhirendranath Thatoi, ${ }^{1}$ Sasanka Choudhury, ${ }^{1}$ and Prabir Kumar Jena Jena ${ }^{2}$ \\ ${ }^{1}$ Department of Mechanical Engineering, ITER, SOA University, Bhubaneswar, Odisha 751030, India \\ ${ }^{2}$ VSSUT, Burla, Odisha 768018, India \\ Correspondence should be addressed to Dhirendranath Thatoi; dhirendrathatoi@soauniversity.ac.in
}

Received 31 May 2014; Accepted 18 November 2014; Published 17 December 2014

Academic Editor: Marc Thomas

Copyright (c) 2014 Dhirendranath Thatoi et al. This is an open access article distributed under the Creative Commons Attribution License, which permits unrestricted use, distribution, and reproduction in any medium, provided the original work is properly cited.

\begin{abstract}
This paper presents a novel hybrid fuzzy logic based artificial intelligence (AI) technique applicable to diagnosis of the crack parameters in a fixed-fixed beam by using the vibration signatures as input. The presence of damage in engineering structures leads to changes in vibration signatures like natural frequency and mode shapes. In the first part of this work, a structure with a failure crack has been analyzed using finite element method (FEM) and retrospective changes in the vibration signatures have been recorded. In the second part of the research work, these deviations in the vibration signatures for the first three mode shapes have been taken as input parameters for a fuzzy logic based controller for calculation of crack location and its severity as output parameters. In the proposed fuzzy controller, hybrid membership functions have been taken. Several fuzzy rules have been identified for prediction of crack depth and location and the results have been compared with finite element analysis. A database of experimental results has also been considered to check the robustness of the fuzzy controller. The results show that predictions for the nondimensional crack location, $\alpha$, deviate $\sim 2.4 \%$ from experimental values and for the nondimensional crack depth, $\delta$, are less than $\sim-2 \%$.
\end{abstract}

\section{Introduction}

Cracks may lead to premature failure in engineering structures. The severity and location of cracks in structures can be identified using nondestructive tests and as an inverse problem using optimization techniques. In the recent past, artificial intelligence methods are found to more efficient in this regard and various efforts have been made.

Shim and Suh [1] presented a method which uses a synthetic artificial intelligence technique, that is, adaptivenetwork-based fuzzy inference system (ANFIS) solved via a hybrid learning algorithm (the back propagation gradient descent and the least-squares method) and continuous evolutionary algorithms (CEAs) solving single objective optimization problems with a continuous function and continuous search space. Ganguli [2] developed a fuzzy logic system (FLS) for ground based health monitoring of a helicopter rotor blade. The structural damage was modelled as a loss of stiffness at the damaged location that can result from delamination. A fuzzy gain tuner to tune the gain in the positive position feedback control to reduce the initial overshoot while still maintaining quick vibration suppression has been presented by $\mathrm{Gu}$ and Song [3]. Jena et al. [4] proposed a differential evolution algorithm for detecting the crack, in which the damage location and severity assessment is formulated as a constraint optimization problem. A novel resonant fuzzy logic controller (FLC) has been developed by Lin and Liu [5] to minimize structural vibration using collocated piezoelectric actuator/sensor pairs. The effectiveness of the new fuzzy control design to a state-of-the-art control scheme is compared through experimental studies.

Chandrashekhar and Ganguli [6] showed that geometric and measurement uncertainty causes considerable problem in the damage assessment. They used Monte Carlo simulation to study the changes in the damage indicator due to uncertainty in the geometric properties of the beam. The results 
obtained from the simulation were used for developing and testing the fuzzy logic system. They addressed the uncertainty associated with the fuzzy logic system for structural damage detection.

Using fuzzy set theory, a computer based crack diagnosis system has been presented by Kim et al. [7]. They used the crack symptoms and characteristics to build rooms for the proposed fuzzy inference system. When they applied the developed methodology to diagnose the crack, the proposed system found the results similar to those obtained by other experts systems. Thatoi et al. [8] have used neural network technique as the key point of their research analysis in which they have proposed cascade forward backpropagation (CFBP) network, feed-forward backpropagation (FFBP) network, and radial basis function (RBF) network for detecting the crack in fixed-fixed beam structures. Saravanan et al. [9] proposed a technique based on vibration signals acquired from operating machines to effectively diagnose the conditions of inaccessible moving components inside the machine. The proposed technique was designed using fuzzy classifier and decision tree to generate the rules automatically from the feature set. The developed fuzzy classifier was tested with representative data and the results were encouraging.

Boutros and Liang [10] developed four condition monitoring indicators for detection of transient and gradual abnormalities using fuzzy logic approach. They successfully tested and validated the fuzzy based technique in two different applications. Wu and Law [11] proposed a novel fuzzy robust wavelet support vector classifier (FRWSVC) based on a wavelet function and developed an adaptive Gaussian particle swarm optimization (AGPSO) algorithm to seek the optimal unknown parameter of the FRWSVC. The results obtained from experimentation were compared with that of the hybrid diagnosis model and were found to be closely matching with each other. Sugumaran and Ramachandran [12] presented the use of decision tree of a fuzzy classifier for selecting best few features that will discriminate the fault condition of the bearing from given trained samples. The vibration signal from a piezoelectric transducer was captured for different types of fault condition of bearing and used to build the fuzzy rules. The results drawn from the fuzzy classifier when compared with results from the experimental analysis were found to be in close proximity.

De Miguel and Blázquez [13] developed a decision making module based on fuzzy logic for model based fault diagnosis applications. A fault detection and isolation system based on the input and output parameters was successfully applied in laboratory equipment to reduce the uncertainties of the output parameter. Nguyen et al. [14] have used an algorithm, which is a combination of an adaptive fuzzy neural structure (AFNS) and an average quantity solution to wavelet transform coefficient (AQWTC) for beam vibration signal. They have discretized the beam into number of elements and subjected it to vibration. They have transformed the vibrating signal into wavelet signal to compare the AQWTC of undamaged status and status at the concerned time. They have utilized these variations to find out elements having strange features in wavelet quantitative analysis, which actually represents the beam damage signs. The robustness of the above approach combining fuzzy neural structure and wavelet transform methods was validated by experiment on measured data sets in a vibrated beam-type steel frame structure. Serhat Erdogan and Gundes Bakir [15] used fuzzy finite element model updating (FFEMU) method for the damage detection problem using the uncertainty caused by the measurement noise in modal parameters by fuzzy numbers. They have determined that reduction in bending stiffness of the elements corresponds to each of the updating parameters of membership functions by minimizing an objective function using a hybrid version of genetic algorithms (GA) and particle swarm optimization method (PSO), which they found to be very efficient in terms of accuracy and robustness. They have also performed a probabilistic analysis using Monte Carlo simulation (MCS) and compared the results with FFEMU method.

Thatoi et al. [16] presented a critical review on various traditional and nontraditional methods used for the crack detection. Beena and Ganguli [17] have developed a new algorithmic approach for structural damage detection based on the fuzzy cognitive map (FCM). They have modelled the structural damage using a continuum mechanics approach as a loss of stiffness at the damaged location and used a finite element model of a cantilever beam to determine the change in the first six beam frequencies due to the structural damage. Then they have fuzzified the measurement deviations due to damage and mapped them to a set of faults using FCM. They have found better results when an unsupervised neural network approach based on Hebbian learning is used to evolve the FCM. They have also validated the results obtained from above techniques by numerical results.

Reddy and Ganguli [18] have proposed Fourier analysis for identification of crack location and depth of a fixedfixed beam. They have also used neural network which is trained to detect the damage location and size using Fourier coefficients as input. They have found that the method for damage detection using Fourier coefficients and neural network has the capability to detect the location and damage size even in the presence of noise parameters satisfactorily and accurately. Zheng et al. [19] have formulated an algorithm based on radial basis function neural network for structural damage identification. For the development of proposed model they have also used fuzzy logic and genetic algorithm. The radial basis function network has been trained with modal frequencies (obtained from finite element analysis) to identify the damage. They have used the composite laminated beam for the analysis. They have found that the developed model is capable of predicting the results within an error of 18 percent.

Sawyer and Rao [20] have presented a generalized methodology for structural fault detection using fuzzy logic. Fuzzy logic and continuum damage mechanics were used by them to process and analyze the uncertainties and complexities of damaged structure considering measurement noise. Pawar and Ganguli [21] have proposed genetic fuzzy system to find the location and extent of damage in beams and helicopter rotor blades. A fuzzy system has been generated with changes in natural frequencies (obtained from finite element analysis of a cantilever beam). The fuzzy rules and 


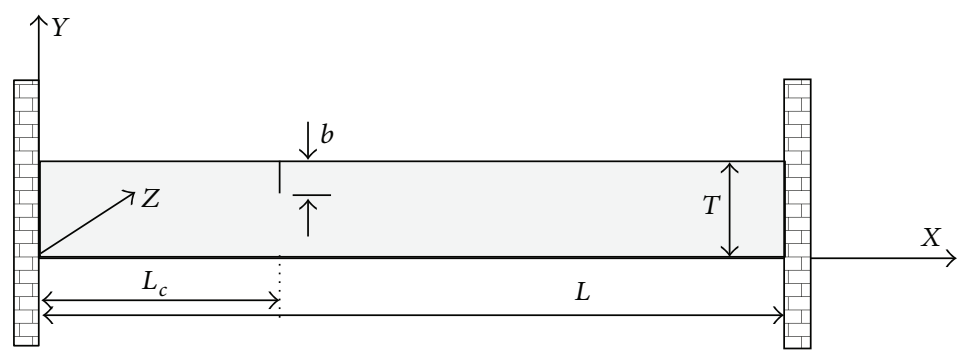

(a)

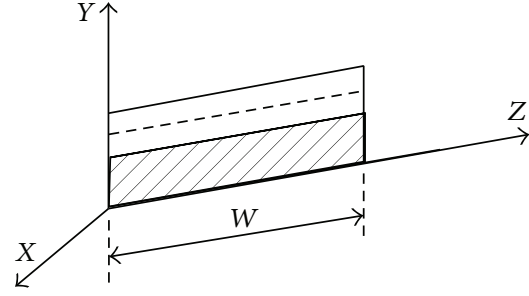

(b)

FIGURE 1: Geometry of the beam: (a) fixed-fixed beam and (b) cross-sectional view of the beam.
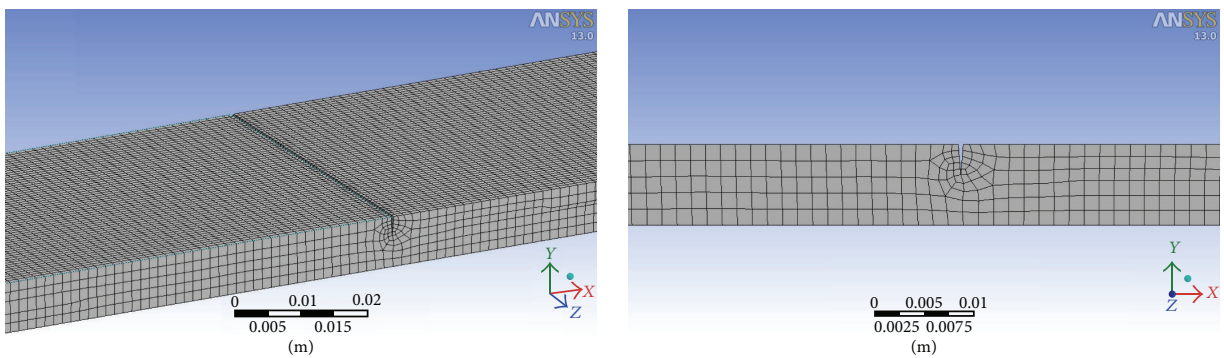

Figure 2: Finite element mesh with mesh zooms of the crack.

membership function are optimized by genetic algorithm. da Silva et al. [22] have proposed fuzzy clustering analysis for the damage detection. They have performed the process through three stages such as principle component analysis (PCA), autoregressive moving average (ARMA), and fuzzy clustering approach. They have used two fuzzy clustering approaches, namely, fuzzy $c$-means (FCM) and Gustafson-Kessel (GK). They have compared the results of both clustering approaches and found that GK clustering approach marginally outperforms the FCM clustering approach. Artificial intelligence methods such as genetic fuzzy system, genetic algorithm, and fuzzy cognitive maps can be applied for damage location and severity. Regarding this kind of methods, several monographs have been presented [23].

From the above discussion it can be concluded that few attempts have been made in using AI technique methods as an alternate method to detect the cracks in a structure. However, in this study, it was found that a hybrid fuzzy system as described in this work is a better alternative to existing approaches. The fuzzy inference system discussed in this paper is validated with numerical and experimental results.

\section{Methodology}

The methodology for using fuzzy interface system includes development of database using finite element method (numerical) and experimental results. The procedure for the finite element analysis and experimental setup used in the present study are presented first and then the methodology for development of fuzzy interface system is discussed.

2.1. Finite Element Analysis. The vibrational analysis of a continuous system by analytical procedures is quite appropriate and less complicated. However, with the introduction of crack in the continuous system the analysis for its vibrational characteristics becomes more complicated. Since the equation of motion of the continuous beam is a partial differential equation supplemented by various initial and boundary conditions, the finite element method (FEM) can be used to translate the complex partial differential equations into linear algebraic equations and hence the mode of solution becomes simpler.

A fixed-fixed beam (Figure 1) of length " $L$," width " $W$," and height " $T$ " with a surface transverse crack of depth " $b$ " taken at a locations " $L_{c}$ " is used for the analysis. The presence of crack introduces a local flexibility. In the present research, ANSYS 13.0 [24] has been used as a tool to model and simulate a beam with and without crack and to monitor the variation in its vibrational characteristics. The beam is modelled using the software Solidworks and is imported within ANSYS for the analysis.

Now, after importing the model file, its geometry has been modified and the entire structure has been discretized into meshes (Figure 2) by using FEM and has been solved for the different modes of vibration. The meshing size should be increased so that it uniformly covers the entire structure. After the model has been properly meshed and solved by using FEM, the different modal frequency values were obtained for a particular combination of crack location and depth. Typical mode shape deflections for the different modes have been shown in Figure 3.

2.2. Experimental Analysis. To validate the current technique used for crack detection an experimental analysis also has been carried out. The experiment has been conducted in two ways. The pictorial view of experimental setup 1 and setup 

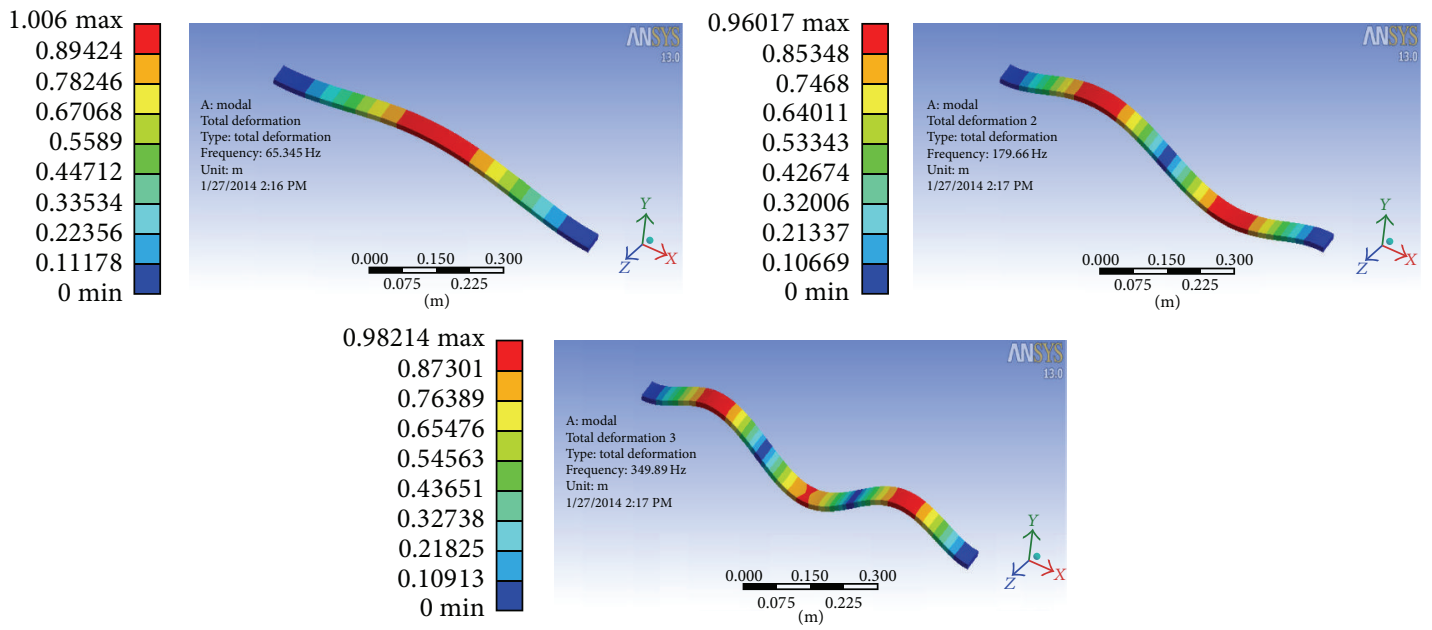

FIgURE 3: Mode shape deflections obtained from ANSYS for 1st, 2nd, and 3rd modes.
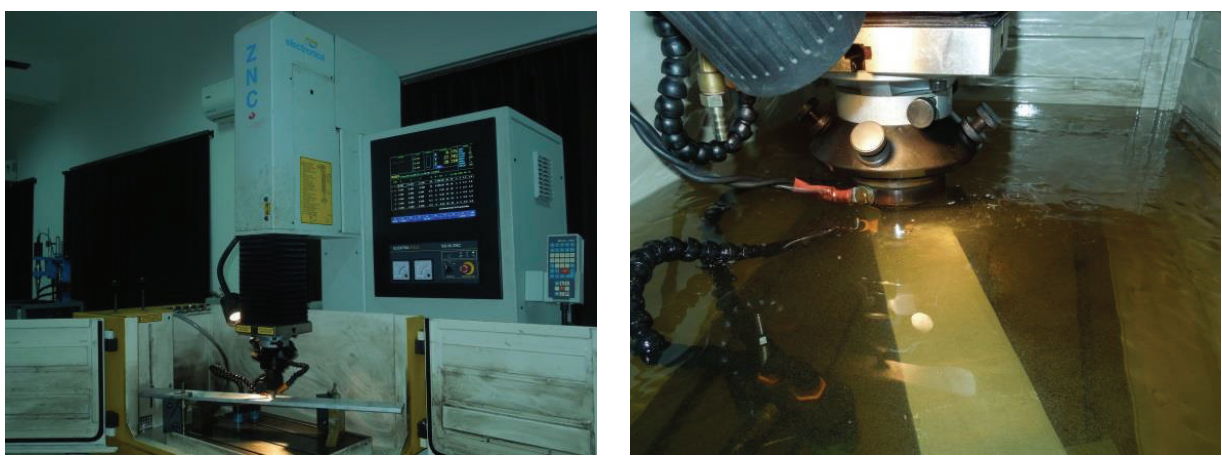

(a)

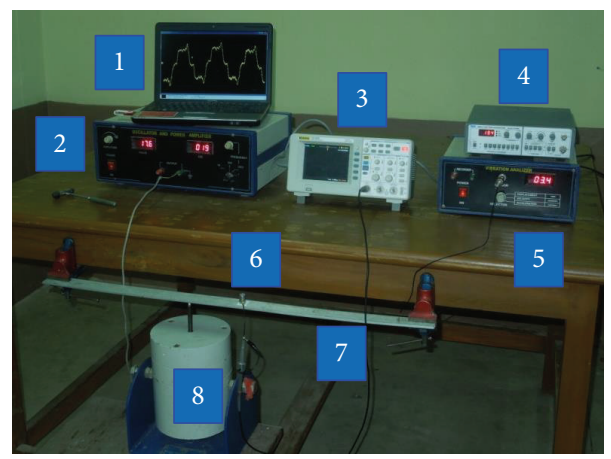

(b)

FIGURE 4: (a) Experimental setup 1: formation of crack using wire cut electrodischarge machine. (b) Experimental setup 2: for measurement of modal parameters ((1) vibration indicator with laptop using PULSE Labshop Software, (2) power amplifier, (3) digital oscilloscope, (4) function generator, (5) vibration analyser, (6) vibration pick-up, (7) fixed-fixed beam, and (8) vibration exciter).

2 is shown in Figure 4. In setup 1, a hairline crack has been developed on the beam using wire cut electric discharge machine (EDM) (manufacturer: Electronica, model: Smart Zone, pulse generator type: MOSFET, Power Supply-3 Phase, AC 415 Volt, $50 \mathrm{~Hz}, 6 \mathrm{KVa}$ ). Lubricant oil has been used as the dielectric for carrying out the process. In the process, a DC
Servomotor has been used to control the $Z$-axis movement of the tool bit. The tool is positioned using incremental linear scale coordinate system. After generating the crack the beam has been processed through setup 2 to obtain the required results. In this setup the cracked fixed-fixed beam is rigidly clamped to the table. Several tests are conducted using 
the experimental setup on aluminum alloy beam specimens $(800 \times 38 \times 6 \mathrm{~mm})$ with the transverse crack used for determining the natural frequencies and mode shapes for different crack locations and crack depths. These specimens have been set to vibrate under 1st, 2nd, and 3rd modes and the corresponding amplitudes are recorded in the vibration indicator using PULSE Labshop Software as well as using a digital oscilloscope. The beam has been excited with a vibration exciter, excited by the signal from the function generator. The signal is amplified by a power amplifier before being fed to the vibration exciter. Experimental results of amplitude of transverse vibration at various locations along the length of the beam have been recorded by positioning the vibration pick-up and tuning the vibration generator at the corresponding resonant frequencies. The material properties of used specimen are determined using standard tests and are presented in Table 1.

The relative natural frequency and relative mode shape difference used in this analysis can be defined as follows:

$$
\begin{aligned}
& \text { Relative natural frequency }\left(\bar{\Delta}_{\mathrm{nf}_{i}, i=1,2,3}\right) \\
& =\frac{\text { Natural frequency of cracked beam }}{\text { Natural frequency of uncracked beam }},
\end{aligned}
$$

Relative mode shape difference $\left(\bar{\Delta}_{\mathrm{m}_{i}, i=1,2,3}\right)$

$=($ Modal amplitude of uncracked beam

- Modal amplitude of cracked beam)

$$
\times(\text { Modal amplitude of uncracked beam })^{-1} \text {. }
$$

\section{Fuzzy Inference System for Crack Detection}

Fuzzy logic is a tool for embedding human structured knowledge (experience, expertise, and heuristic). According to Zadeh, the father of fuzzy logic, "fuzzy logic may be viewed as a bridge over the excessively wide gap between the precision of classical crisp logic and the imprecision of both the real world and its human interpretation" [25]. Fuzzy logic attempts to model the way of reasoning that goes in the human brain. Almost all of human experience is stored in the form of the if-then rules. Human reasoning is pervasively approximate, nonquantitative, linguistic, and dispositional. Fuzzy logic can be explained in the following steps.

3.1. Fuzzy Set. A fuzzy set, as the name implies, is a set without a crisp boundary. That is, the transition from "belongs to a set" to does "not belong to a set" is gradual and this smooth transition is characterized by membership functions that give fuzzy sets flexibility in modelling commonly used linguistic expressions. A membership function assigns a membership grade to each element in the set under consideration, which is a value in the interval $[0,1]$.

3.2. Membership Function. The basic structure of a fuzzy interface system consists of three components: a rule base, which contains a selection of fuzzy rules, a database, which defines the membership functions used in the fuzzy rules, and a reasoning mechanism, which performs the interface procedure. The membership function $\mu A(x)$ describes the membership of the elements $x$ of the base set $X$ in fuzzy set $A$, whereby $\mu A(x)$ a large class of function can be taken. Reasonable functions are often piecewise linear functions, such as triangular or trapezoidal functions. The value for the membership function can be taken in the interval $[0,1]$. When the functions are nonlinear the Gaussian membership functions are considered for the smooth operation. However, it would be prudent to consider different membership functions and their contributions for better approximations. Hence in the present analysis hybrid membership function (combination of triangular, Gaussian, and trapezoidal) has been used. The membership functions used for the inputs as well as outputs are shown in Figure 5. The linguistic terms used for the membership functions are explained in Table 2.

3.3. Fuzzy Logic. In crisp logic, the truth values acquired by proposition or predicates are 2 -valued, namely, true and false, which may be treated numerically as equivalent to 1 and 0 , respectively. However, in fuzzy logic, truth values are multivalued such as absolutely false, partly false, partly true, and very true and are made to numerically vary between zero and one.

3.4. Fuzzy Linguistic Variables. Just like an algebraic variable takes numbers as values, a linguistic variable takes words or sentences as values. The set of values that it can take is called its term set. Each value in the term set is a fuzzy variable defined over a base variable. The base variable defines the universe of discourse for all the fuzzy variables. In short the hierarchy is as follows: linguistic variable $\rightarrow$ fuzzy variable $\rightarrow$ base variable.

3.5. Fuzzy If-Then Rule. A fuzzy if-then rule (also known as fuzzy rule, fuzzy implication, or fuzzy conditional statement) assumes the form "if $x$ is $A$ then $y$ is $B$," where $A$ and $B$ are linguistic values defined by fuzzy sets on universes of discourse $x$ and $y$, respectively. Often " $x$ is $A$ " is called the antecedent or premise, while " $y$ is $B$ " is called the consequence or conclusion. The linguistic terms used are shown in Table 2 and out of $10^{6}$ (number of membership functions to the power of number of inputs) fuzzy rules some of the fuzzy rules have been stated in Table 3 .

Table 2 describes the name of the membership functions used, linguistic terms, and their description. In this table the first letter symbol "L" stands for lower range, "M" stands for medium range, and " $H$ " stands for higher range for various membership functions and the second letter " $\mathrm{F}$ " and "M" stand for the frequency and mode shape, respectively. By combining the linguistic terms used for the various membership functions the fuzzy rules are generated. Out of several rules, some of the rules are mentioned in Table 3.

3.6. Fuzzy Controller. Fuzzy logic controllers are based on the combination of fuzzy set theory which is controlled by rules instead of equations. The collections of rules are known as 


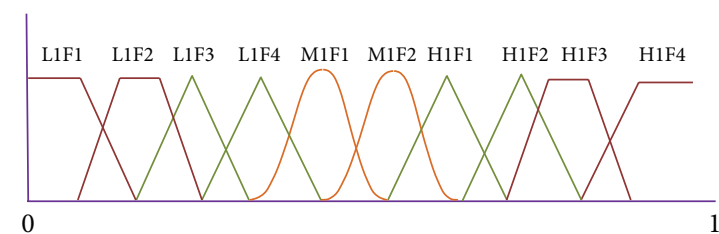

(a)

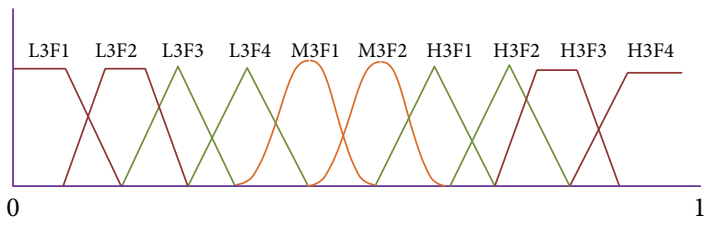

(c)

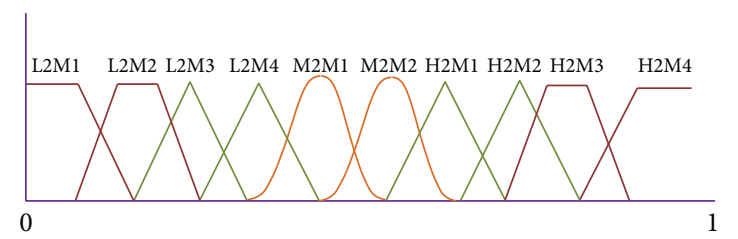

(e)

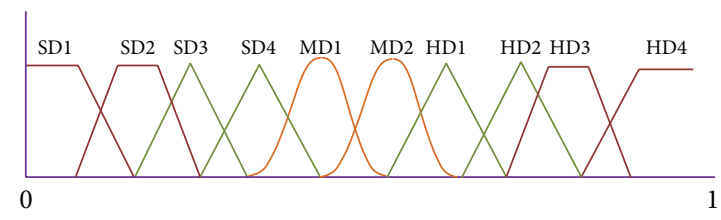

(g)

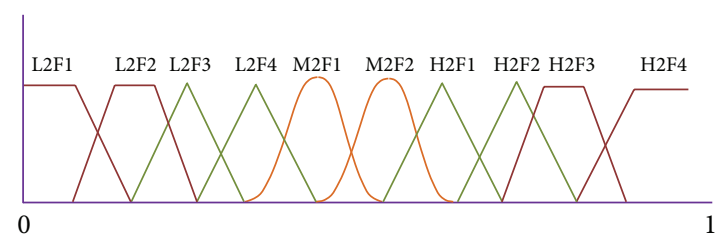

(b)

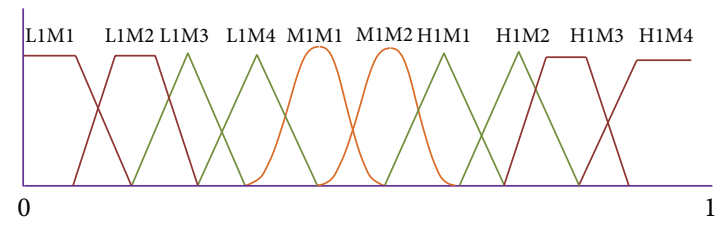

(d)

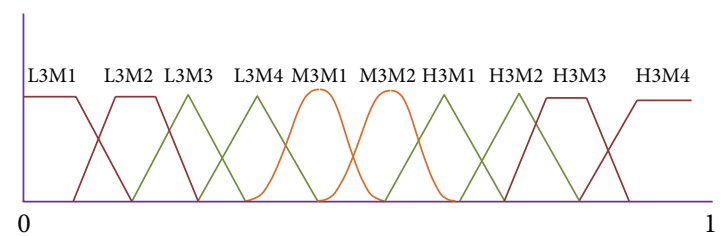

(f)

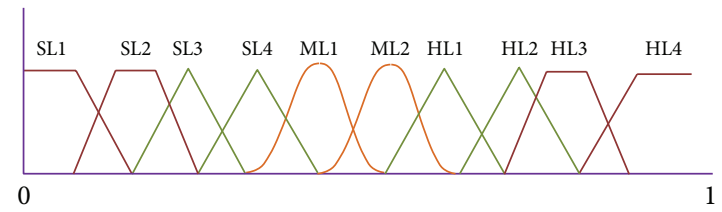

(h)

FIGURE 5: Hybrid membership function showing (a) relative first natural frequency, (b) relative second natural frequency, (c) relative third natural frequency, $(\mathrm{d})$ relative mode shape difference of first mode, (e) relative mode shape difference of second mode, (f) relative mode shape difference of third mode, $(\mathrm{g})$ relative crack depth, and $(\mathrm{h})$ relative crack location.

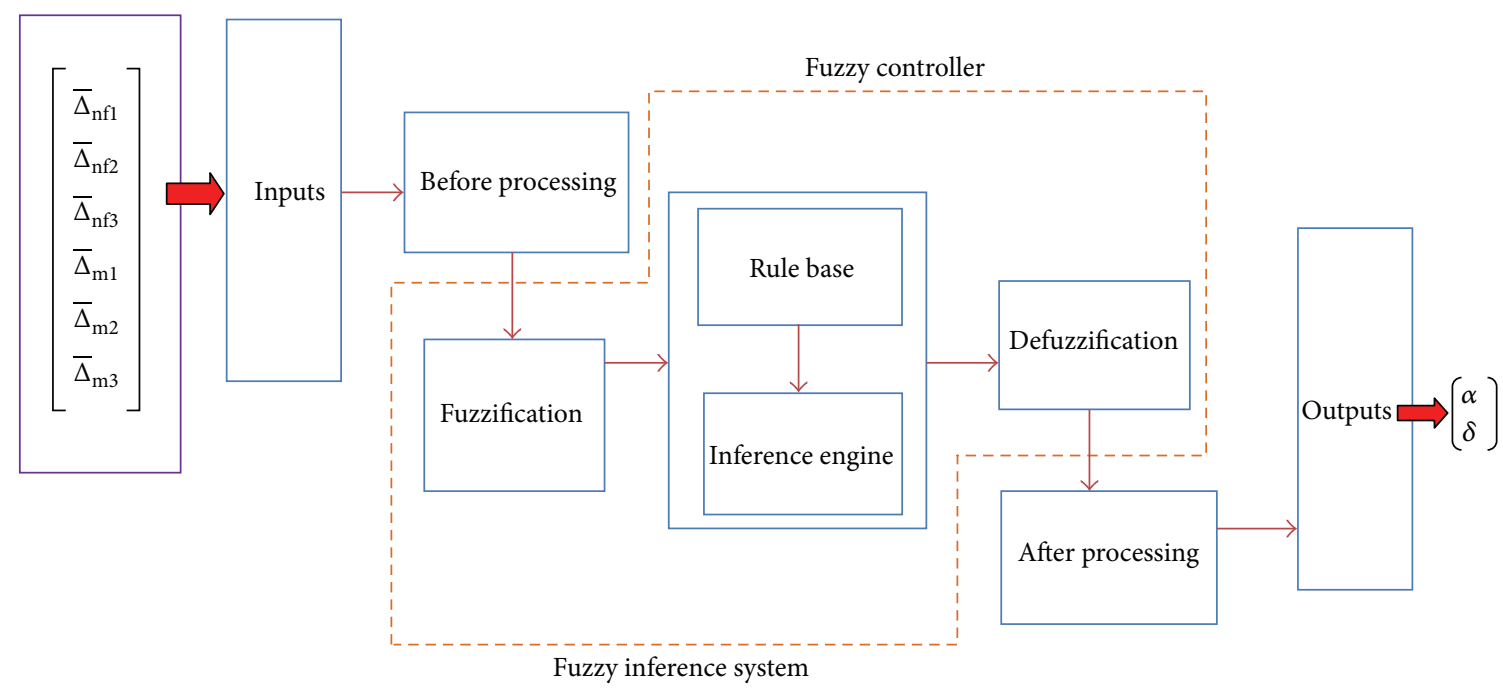

FiguRE 6: Fuzzy controller used in the present study.

TABLE 1: Material properties of aluminium alloy, 2014- $\mathrm{T}_{4}$.

\begin{tabular}{lccccc}
\hline $\begin{array}{l}\text { Young's modulus }(E) \\
\text { GPa }\end{array}$ & $\begin{array}{c}\text { Density }(\rho) \\
\mathrm{gm} / \mathrm{cc}\end{array}$ & Poisson's ratio $(\mu)$ & $\begin{array}{c}\text { Length }(L) \\
\mathrm{Mm}\end{array}$ & $\begin{array}{c}\text { Width }(W) \\
\mathrm{mm}\end{array}$ \\
\hline 72.4 & 2.8 & 0.33 & 800 & $\begin{array}{c}\text { Depth }(T) \\
\mathrm{mm}\end{array}$ \\
\hline
\end{tabular}



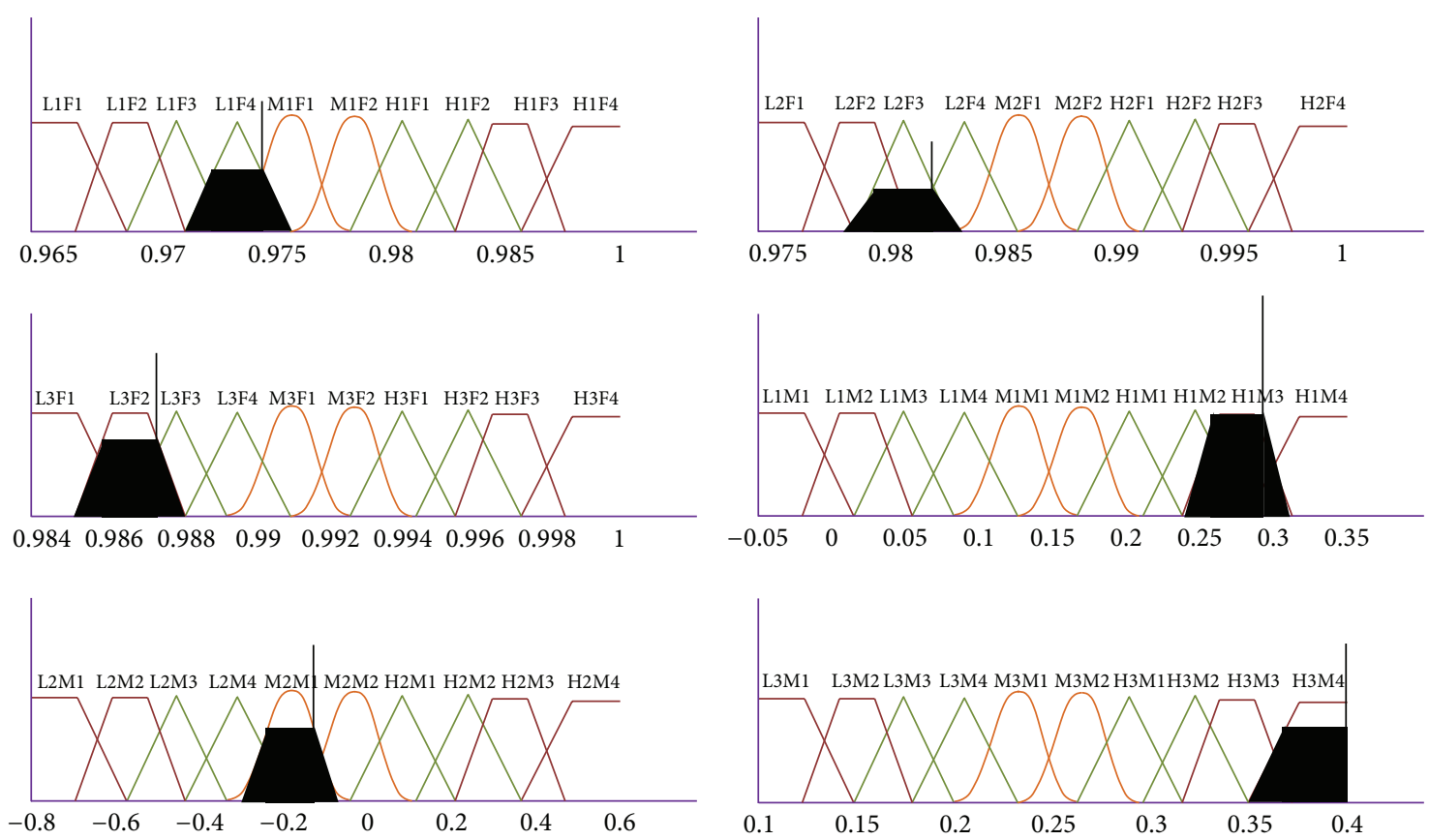

(a)
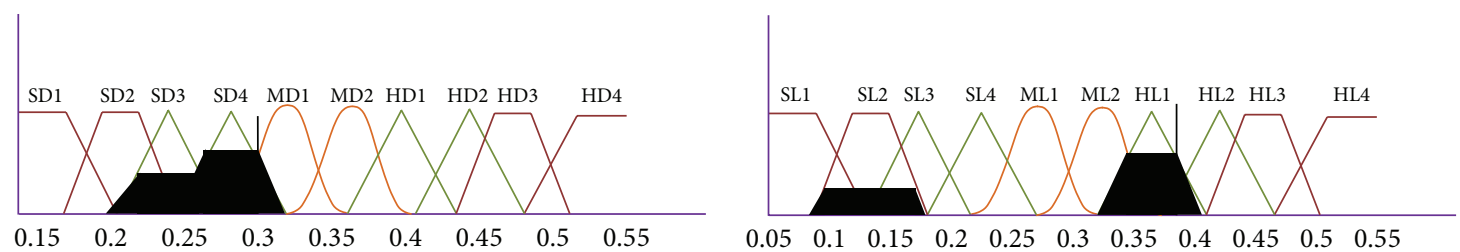

(b)

FIGURE 7: (a) Activation of fuzzy rule inputs. (b) Activation of fuzzy rule generation of outputs.

the rule base usually in the form of "if-then-else" statements. The "if" part is known as antecedent and the "then" part is the consequent. The antecedents are connected with simple Boolean functions like AND, OR, NOT, and so forth [26]. Figure 6 outlines the architecture of fuzzy logic controller, which is used in the current analysis. Once all the rules have been defined based on the application, the control process starts with the computation of the rule consequences. The computation of the rule consequences takes place within the computational unit. Finally, the fuzzy set is defuzzified into one crisp control action using the defuzzification module (7a) and (7b).

A typical activation of fuzzy rules of inputs and generation of outputs is shown in Figures 7(a) and 7(b), respectively. In Figure 7 the activation process has been framed out by taking the second rule of Table 3 .

3.7. Fuzzy Mechanism Used for Crack Detection. Based on the above fuzzy subsets, the rules of fuzzy inference system are defined in a general form. A fuzzy controller has been developed with six input parameters and two output parameters.

The six inputs are as follows:

(1) nondimensional first natural frequency $=\bar{\Delta}_{\mathrm{nf}_{1}}$,
(2) nondimensional second natural frequency $=\bar{\Delta}_{\mathrm{nf}_{2}}$,

(3) nondimensional third natural frequency $=\bar{\Delta}_{\mathrm{nf}_{3}}$,

(4) nondimensional first mode shape difference $=\bar{\Delta}_{\mathrm{m}_{1}}$,

(5) nondimensional second mode shape difference $=$ $\bar{\Delta}_{\mathrm{m}_{2}}$,

(6) nondimensional third mode shape difference $=\bar{\Delta}_{\mathrm{m}_{3}}$.

The two outputs are as follows:

(1) nondimensional crack location $=\alpha$,

(2) nondimensional crack depth $=\delta$ :

$$
\begin{aligned}
& \text { If }\left(\bar{\Delta}_{\mathrm{nf}_{1}} \text { is } \bar{\Delta}_{\mathrm{nf}_{i}}, \bar{\Delta}_{\mathrm{nf}_{2}} \text { is } \bar{\Delta}_{\mathrm{nf}_{\mathrm{j}}}, \bar{\Delta}_{\mathrm{nf}_{3}} \text { is } \bar{\Delta}_{\mathrm{nf}_{k}},\right. \\
& \left.\bar{\Delta}_{\mathrm{m}_{1}} \text { is } \bar{\Delta}_{\mathrm{m}_{\ell}}, \bar{\Delta}_{\mathrm{m}_{2}} \text { is } \bar{\Delta}_{\mathrm{m}_{m}} \text {, and tmd is } \bar{\Delta}_{\mathrm{m}_{n}}\right)
\end{aligned}
$$

then non-dimensional crack location is $\alpha_{i j k l m n}$ and non-dimensional crack depth is $\delta_{i j k l m n}$, 

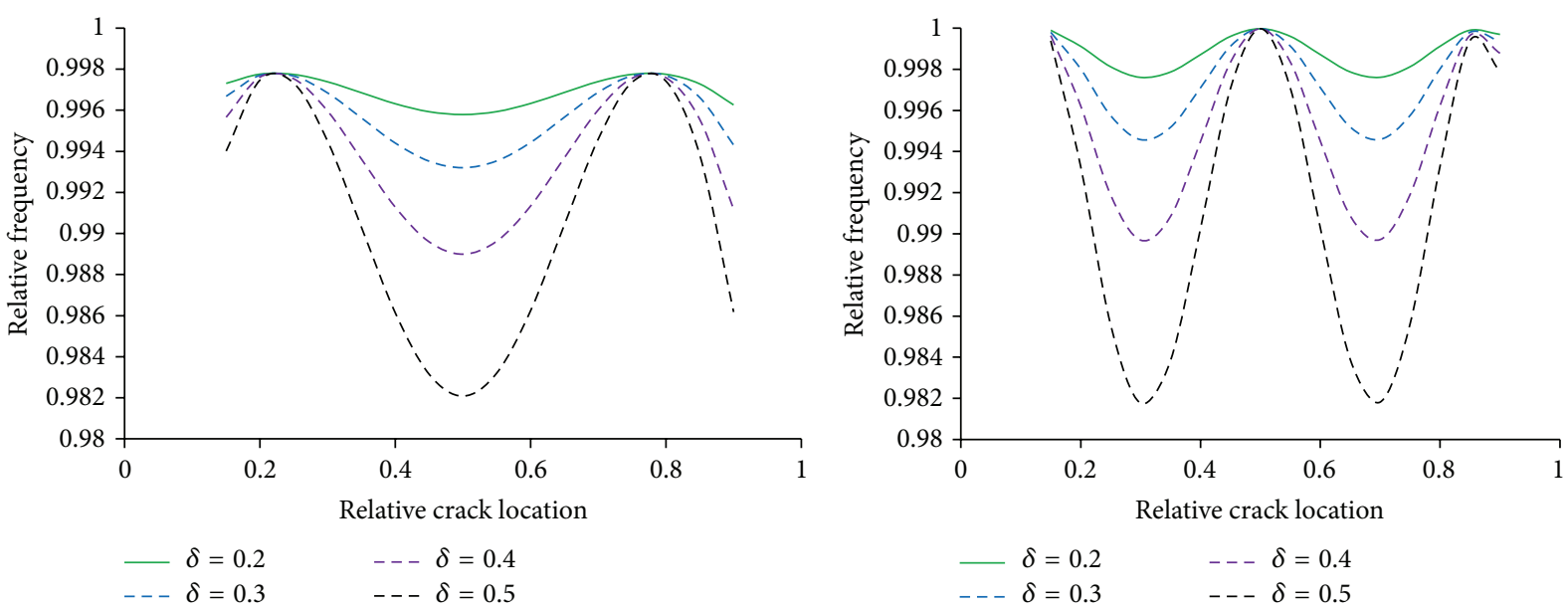

(a)

(b)

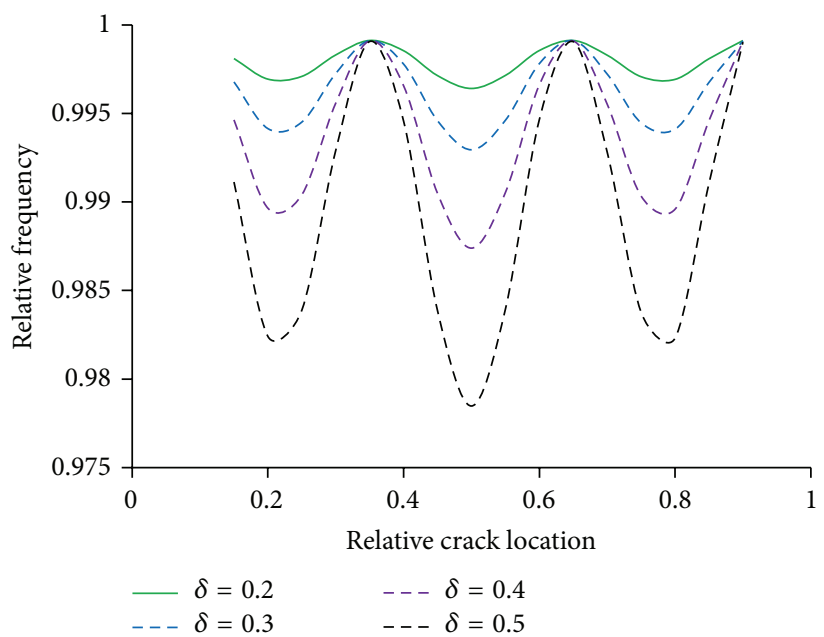

(c)

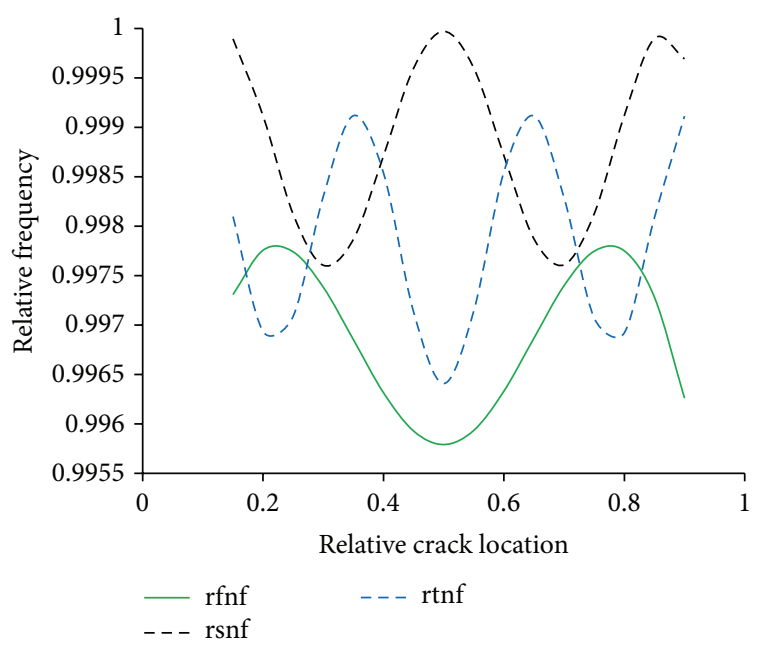

(d)

FIGURE 8: (a) Frequency response plot at various crack locations for the 1st mode of vibration, (b) frequency response plot at various crack locations for the 2nd mode of vibration, (c) frequency response plot at various crack locations for the 3rd mode of vibration, and (d) comparison of frequency response for three mode shapes.

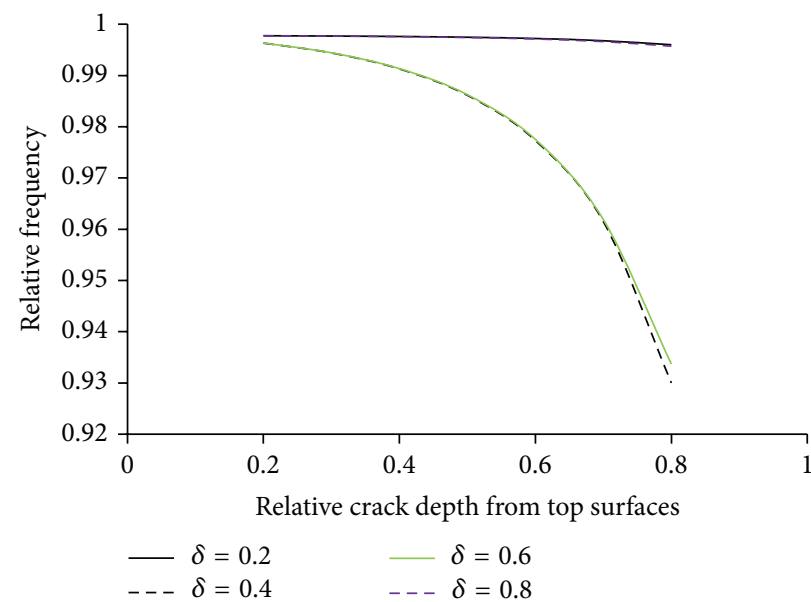

FIGURE 9: Frequency response plot for various crack depths with a given crack location for the 1st, 2nd, and 3rd modes of vibration. 


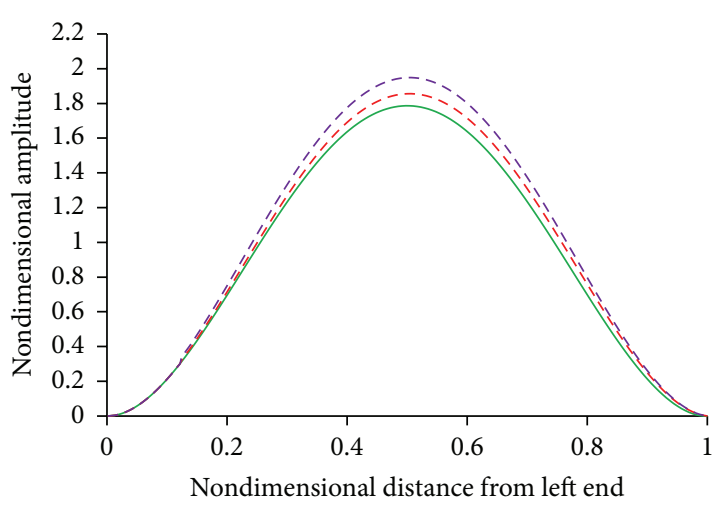

(a)

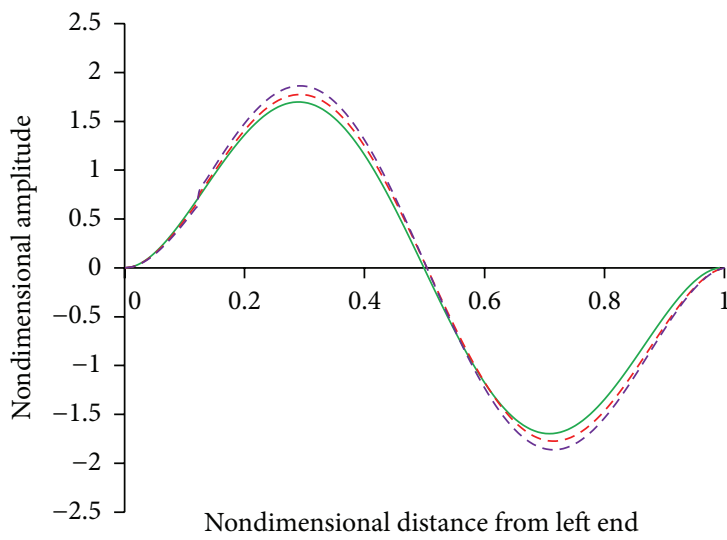

(c)

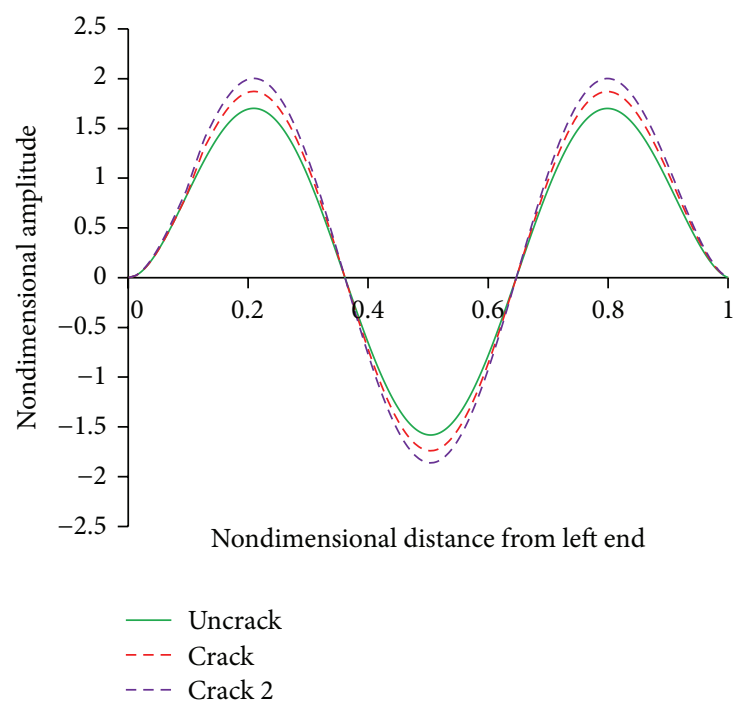

(e)

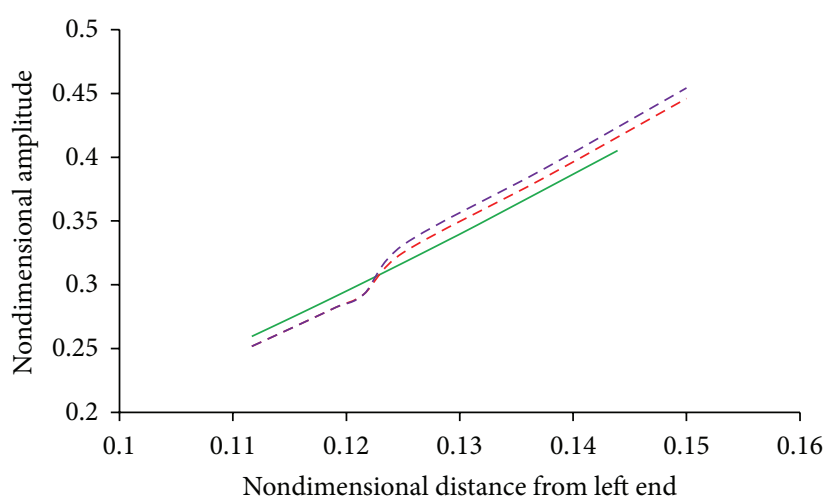

(b)

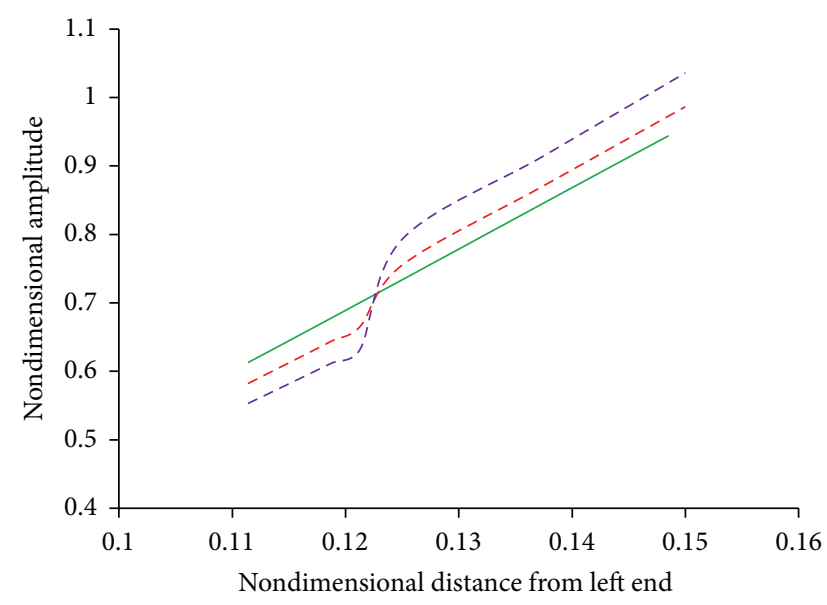

(d)

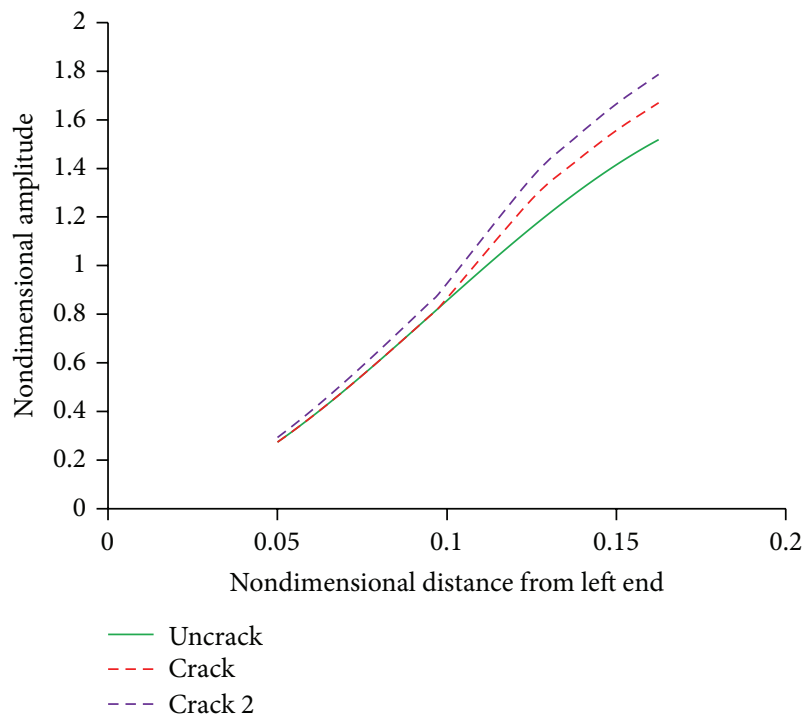

(f)

Figure 10: (a) Nondimensional amplitude versus nondimensional distance from left end (1st mode of vibration), (b) magnified view of crack position in 1st mode of vibration, (c) nondimensional amplitude versus nondimensional distance from left end (2nd mode of vibration), (d) magnified view of crack position in 2nd mode of vibration, (e) nondimensional amplitude versus nondimensional distance from left end (3rd mode of vibration), and (f) magnified view of crack position in 3rd mode of vibration. 


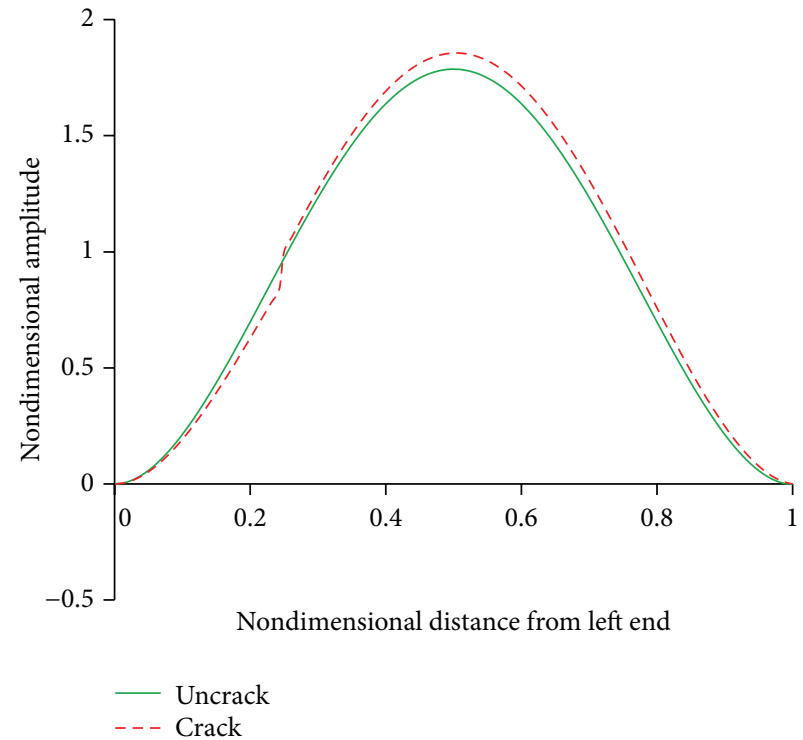

(a)

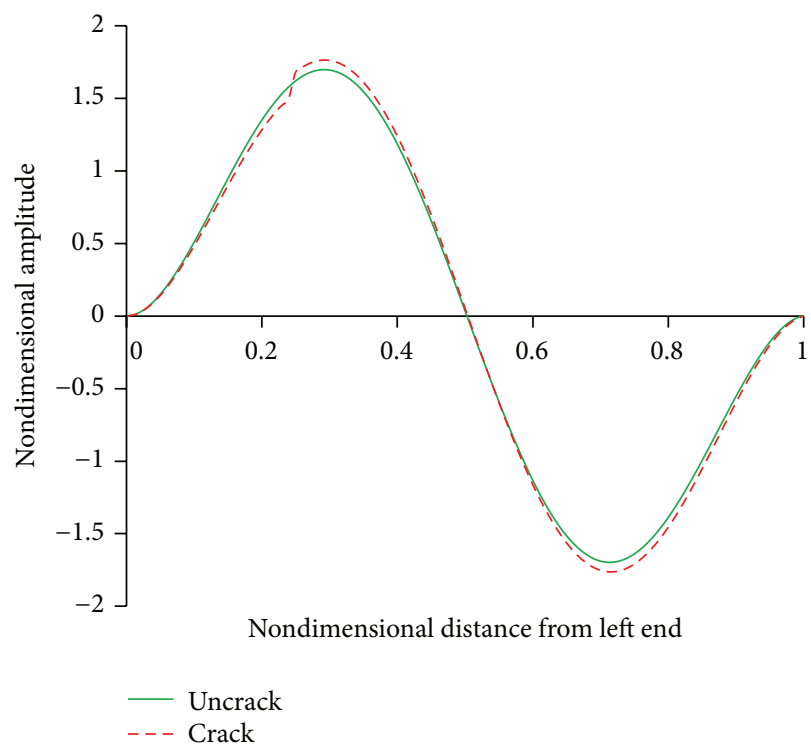

(b)

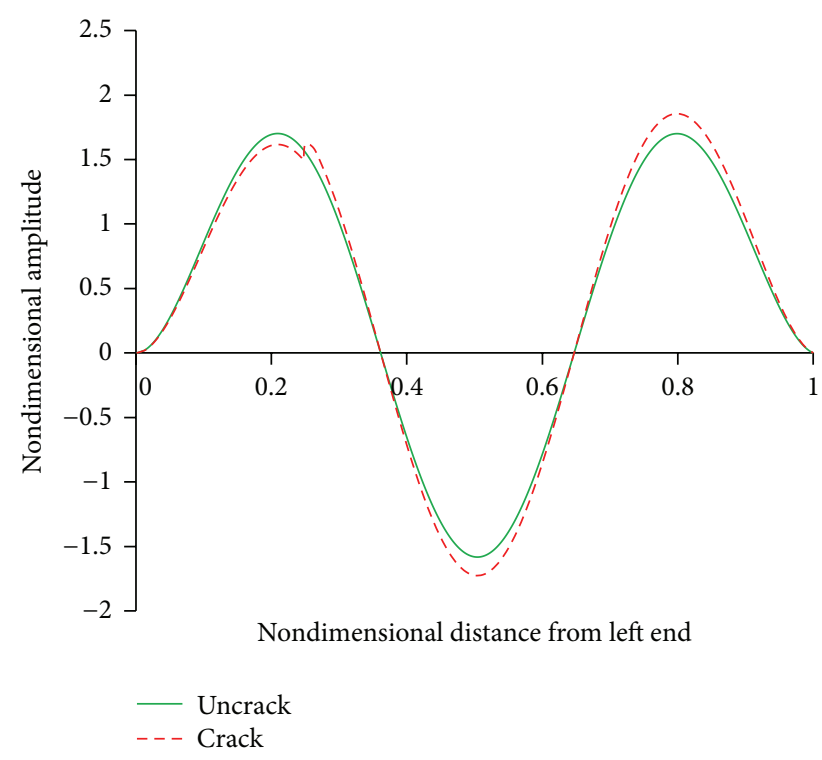

(c)

Figure 11: (a) Nondimensional amplitude versus nondimensional distance from left end (1st mode of vibration), (b) nondimensional amplitude versus nondimensional distance from left end (2nd mode of vibration), and (c) nondimensional amplitude versus nondimensional distance from left end (3rd mode of vibration).

where $i, \ldots, n$ varies from 1 to 10 . This range can be attributed to the fact that $\bar{\Delta}_{\mathrm{nf}_{1}}, \bar{\Delta}_{\mathrm{nf}_{2}}, \bar{\Delta}_{\mathrm{nf}_{3}}, \bar{\Delta}_{\mathrm{m}_{1}}, \bar{\Delta}_{\mathrm{m}_{2}}$, and $\bar{\Delta}_{\mathrm{m}_{3}}$ (first natural frequency, second natural frequency, third natural frequency, first mode shape, second mode shape, and third mode shape) have ten membership functions (lower range- 4 in numbers, medium range- 2 in numbers, and higher range- 4 in numbers) each.

From expression (2), two set of rules can be written as

$$
\text { If }\left(\bar{\Delta}_{\mathrm{nf}_{1}} \text { is } \bar{\Delta}_{\mathrm{nf}_{i}}, \bar{\Delta}_{\mathrm{nf}_{2}} \text { is } \bar{\Delta}_{\mathrm{nf}_{j}}, \bar{\Delta}_{\mathrm{nf}_{3}} \text { is } \bar{\Delta}_{\mathrm{nf}_{k}}\right. \text {, }
$$

$$
\left.\bar{\Delta}_{\mathrm{m}_{1}} \text { is } \bar{\Delta}_{\mathrm{m}_{\ell}}, \bar{\Delta}_{\mathrm{m}_{2}} \text { is } \bar{\Delta}_{\mathrm{m}_{m}} \text {, and } \bar{\Delta}_{\mathrm{m}_{3}} \text { is } \bar{\Delta}_{\mathrm{m}_{n}}\right)
$$

then $\delta$ is $\delta_{i j k l m n}$,

$$
\begin{aligned}
& \text { If }\left(\bar{\Delta}_{\mathrm{nf}_{1}} \text { is } \bar{\Delta}_{\mathrm{nf}_{i}}, \bar{\Delta}_{\mathrm{nf}_{2}} \text { is } \bar{\Delta}_{\mathrm{nf}_{j}}, \bar{\Delta}_{\mathrm{nf}_{3}} \text { is } \bar{\Delta}_{\mathrm{nf}_{k}},\right. \\
& \left.\bar{\Delta}_{\mathrm{m}_{1}} \text { is } \bar{\Delta}_{\mathrm{m}_{\ell}}, \bar{\Delta}_{\mathrm{m}_{2}} \text { is } \bar{\Delta}_{\mathrm{m}_{m}} \text {, and } \bar{\Delta}_{\mathrm{m}_{3}} \text { is } \bar{\Delta}_{\mathrm{m}_{n}}\right)
\end{aligned}
$$

then $\alpha$ is $\alpha_{i j k l m n}$. 


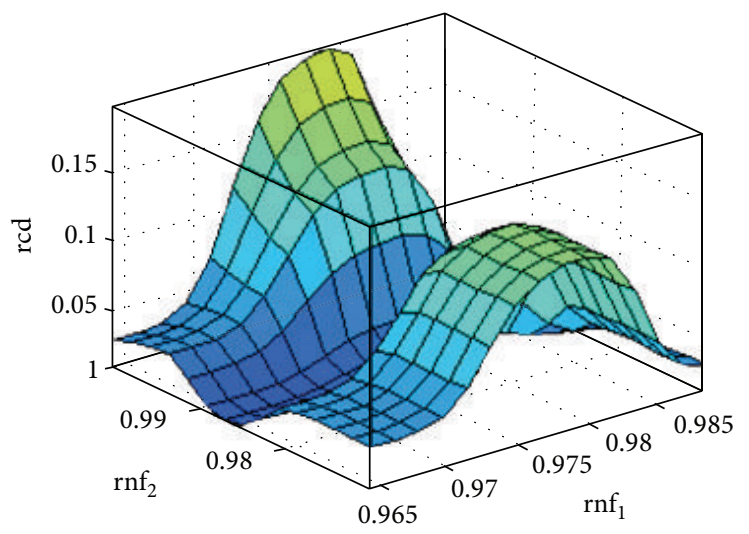

(a) rcd versus $\operatorname{rnf}_{1}$ and $\mathrm{rnf}_{2}$

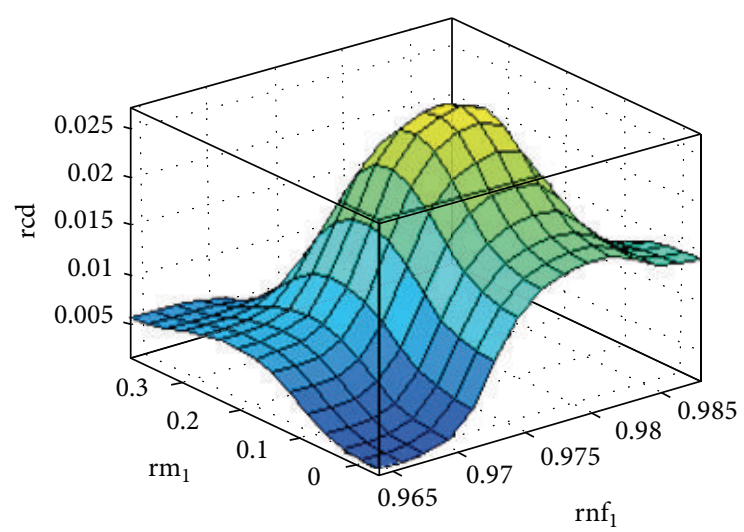

(c) rcd versus $\mathrm{rnf}_{1}$ and $\mathrm{rm}_{1}$

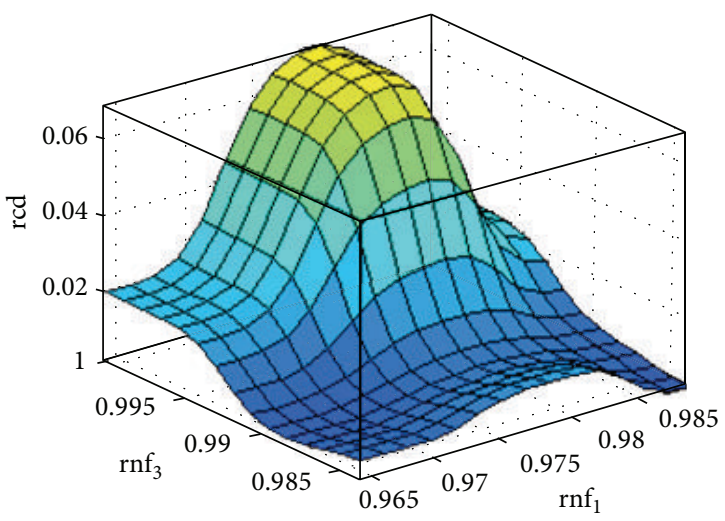

(b) rcd versus $\operatorname{rnf}_{1}$ and $\operatorname{rnf}_{3}$

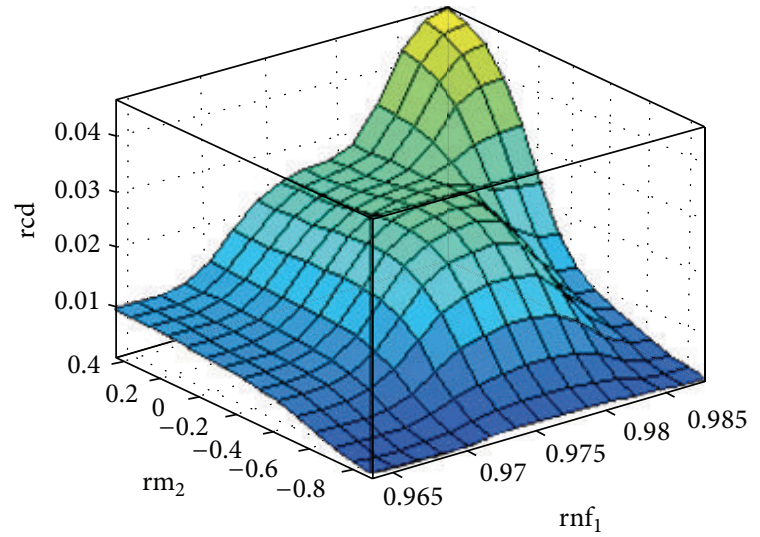

(d) rcd versus $\mathrm{rnf}_{1}$ and $\mathrm{rm}_{2}$

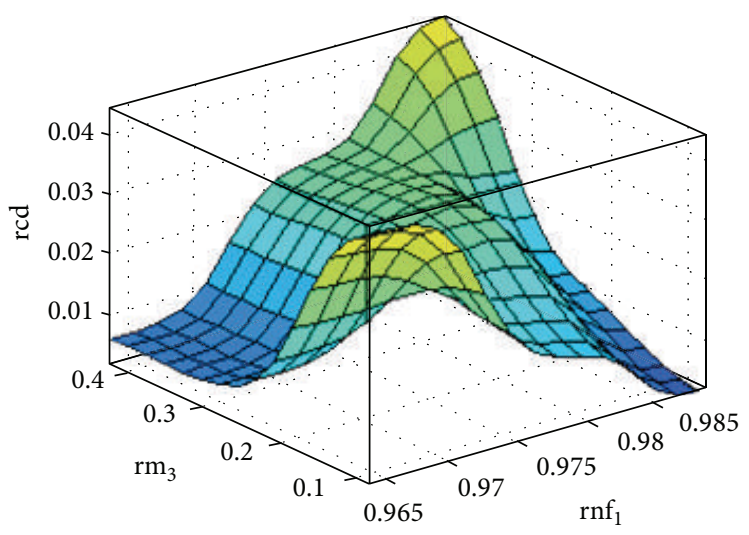

(e) rcd versus $\mathrm{rnf}_{1}$ and $\mathrm{rm}_{3}$

FIGURE 12: Surface plots for relative crack depth versus relative natural frequency $\left(\mathrm{rnf}_{i=1,2,3}\right)$ and relative mode shape differences $\left(\mathrm{rm}_{i=1,2,3}\right)$.

According to the usual fuzzy logic control method [26], a factor $W_{i j k l m n}$ is defined for the rules as follows:

$$
\begin{aligned}
W_{i j k l m n}= & \mu_{\text {fnf }_{i}}\left(\text { freq }_{i}\right) \Lambda \mu_{\text {snf }_{j}}\left(\text { freq }_{j}\right) \Lambda \mu_{\text {tnf }_{k}}\left(\text { freq }_{k}\right) \\
& \times \Lambda \mu_{\text {fmd }_{l}}\left(\text { moddif }_{l}\right) \Lambda \mu_{\text {smd }_{m}}\left(\text { moddif }_{m}\right) \\
& \times \Lambda \mu_{\text {tmd }_{n}}\left(\text { moddif }_{n}\right),
\end{aligned}
$$

where $\mathrm{freq}_{i}$, freq $_{j}$, and freq $\mathrm{q}_{k}$ are the first, second, and third nondimensional frequencies and $\operatorname{moddif}_{l}, \operatorname{moddif}_{m}$, and moddif $_{n}$ are the first, second, and third nondimensional mode shape differences of the fixed-fixed beam with crack, respectively. By applying the composition rule of inference [25], the membership values of the nondimensional crack location $(\alpha)$ and nondimensional crack depth $(\delta)$ can be computed as

$\mu_{\text {rcl }_{i j k l m n}}($ location $)=W_{i j k l m n} \Lambda \mu_{\text {rcl }_{i j k l m n}}$ (location) $\quad \forall_{\text {length }} \in \alpha$, 


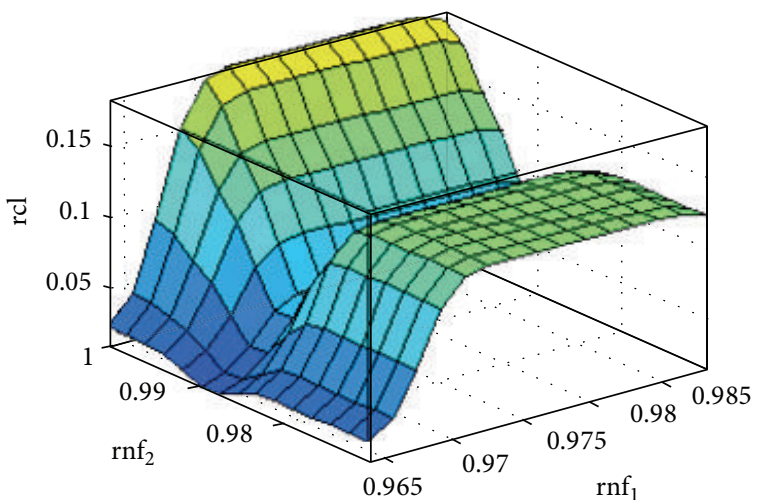

(a) $\mathrm{rcl}$ versus $\mathrm{rnf}_{1}$ and $\mathrm{rnf}_{2}$

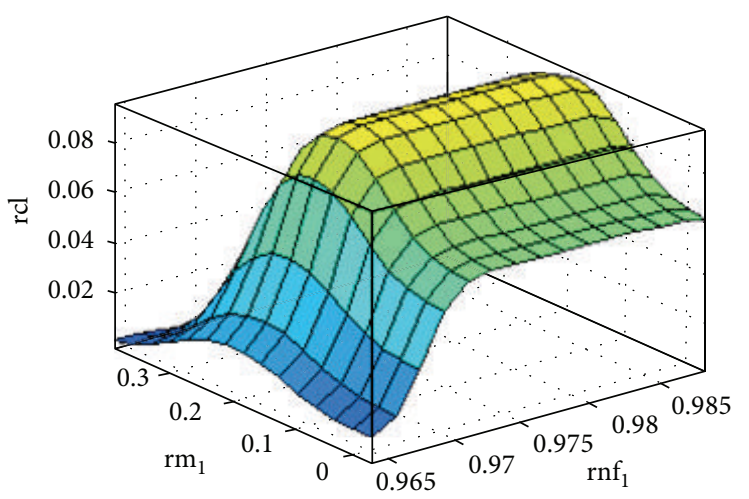

(c) $\mathrm{rcl}$ versus $\mathrm{rnf}_{1}$ and $\mathrm{rm}_{1}$

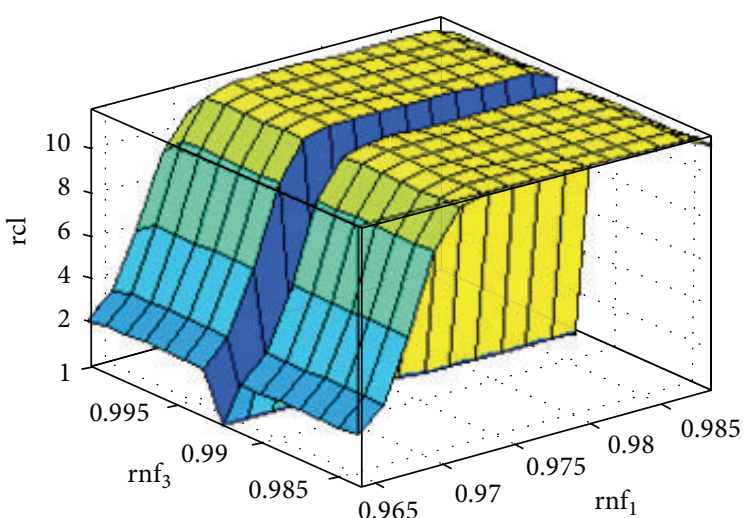

(b) $\mathrm{rcl}$ versus $\mathrm{rnf}_{1}$ and $\mathrm{rnf}_{3}$

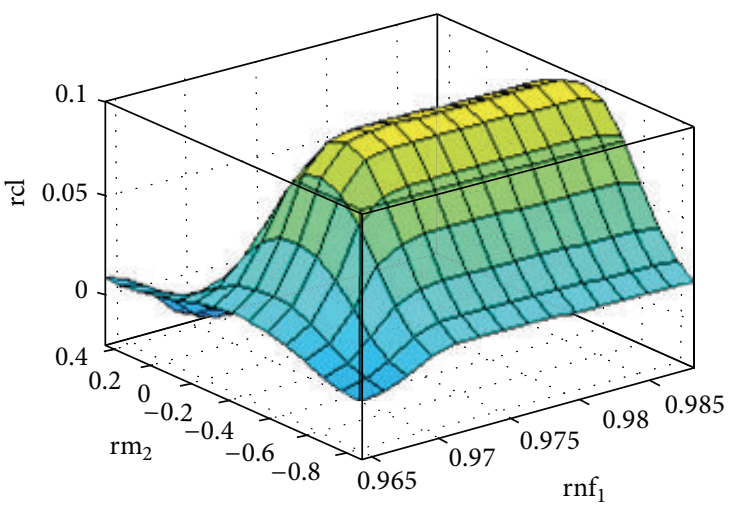

(d) $\mathrm{rcl}$ versus $\mathrm{rnf}_{1}$ and $\mathrm{rm}_{2}$

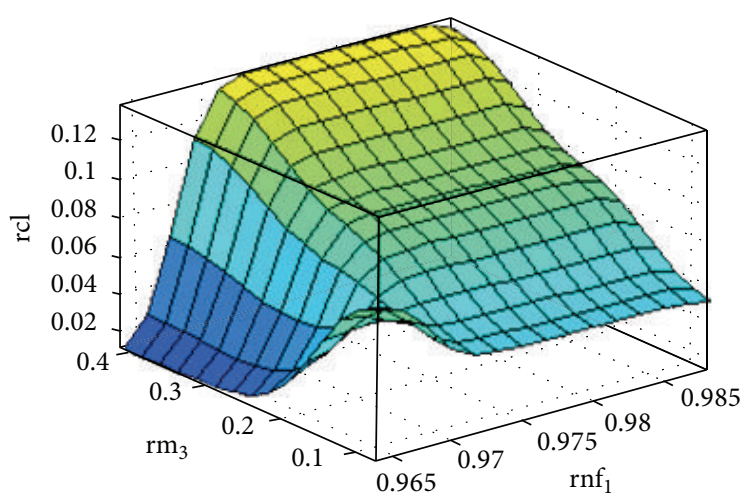

(e) $\mathrm{rcl}$ versus $\mathrm{rnf}_{1}$ and $\mathrm{rm}_{3}$

FIGURE 13: Surface plots for relative crack location versus relative natural frequency $\left(\mathrm{rnf}_{i=1,2,3}\right)$ and relative mode shape differences $\left(\mathrm{rm}_{i=1,2,3}\right)$.

$$
\mu_{\mathrm{rcd}_{i j k l m n}}(\text { depth })=W_{i j k l m n} \Lambda \mu_{\mathrm{rcd}_{i j k l m n}}(\text { depth }) \quad \forall_{\text {depth }} \in \delta .
$$

The overall conclusion by combining the outputs of all the fuzzy rules can be written as follows:

$$
\begin{aligned}
& \mu_{\mathrm{rcl}} \text { (location) } \\
& =\mu_{\mathrm{rcl}_{11111}} \text { (location) } \vee \cdots \vee \mu_{\mathrm{rcl}_{i j k l m n}} \text { (location) } \\
& \quad \vee \cdots \vee \mu_{\mathrm{rcl}_{1010101010} 10} \text { (location), }
\end{aligned}
$$

$$
\begin{aligned}
& \mu_{\mathrm{rcd}}(\text { depth }) \\
& =\mu_{\mathrm{rcd}_{11111}}(\text { depth }) \vee \cdots \vee \mu_{\mathrm{rcd}_{i j k l m n}}(\text { depth }) \\
& \quad \vee \cdots \vee \mu_{\mathrm{rcd}_{1010101010}}(\text { depth }) .
\end{aligned}
$$

The crisp values of nondimensional crack location $(\alpha)$ and nondimensional crack depth $(\delta)$ are computed using the centre of gravity method [25] as follows:

Non-dimensional crack location, $\alpha$

$$
=\frac{\left.\left.\int \text { (location }\right) \cdot \mu_{\mathrm{rcl}} \text { (location }\right) \cdot d \text { (location) }}{\left.\int \mu_{\mathrm{rcl}}(\text { location }) \cdot d \text { (location }\right)},
$$




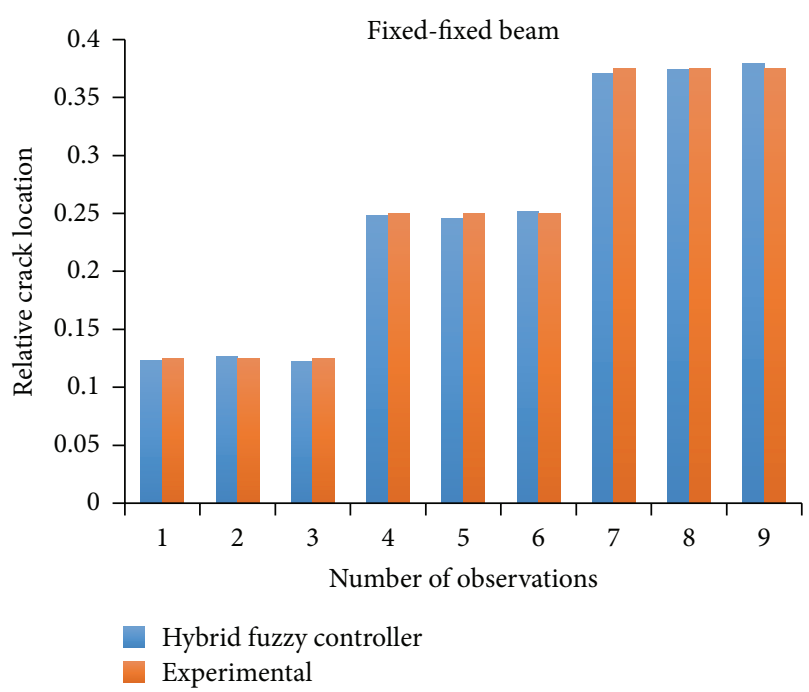

(a)

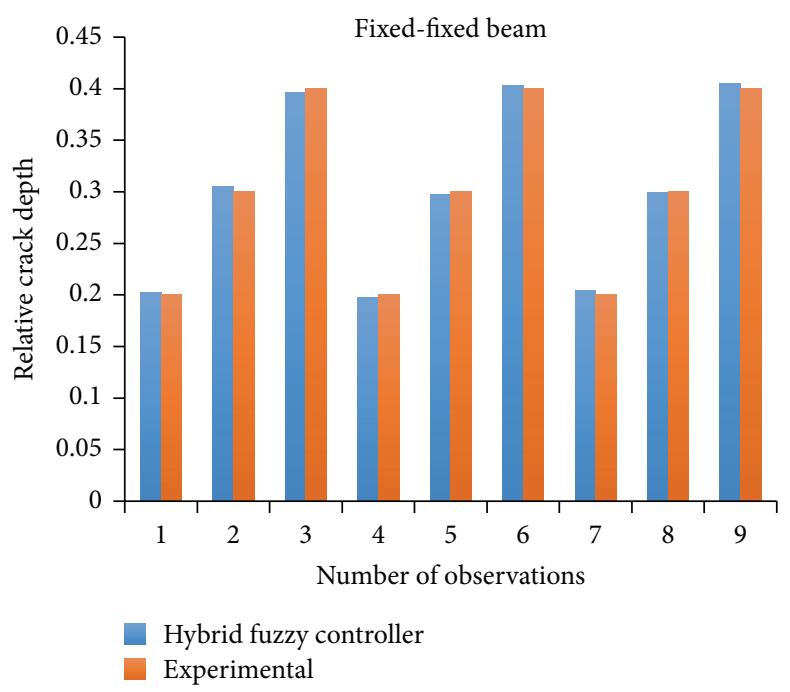

(b)

Figure 14: (a) Comparison of results of relative crack location. (b) Comparison of results of relative crack depth.

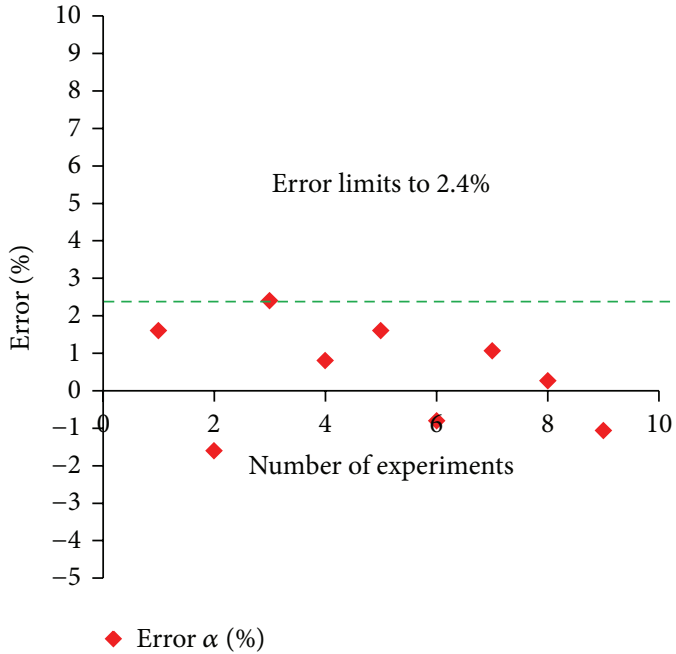

(a)

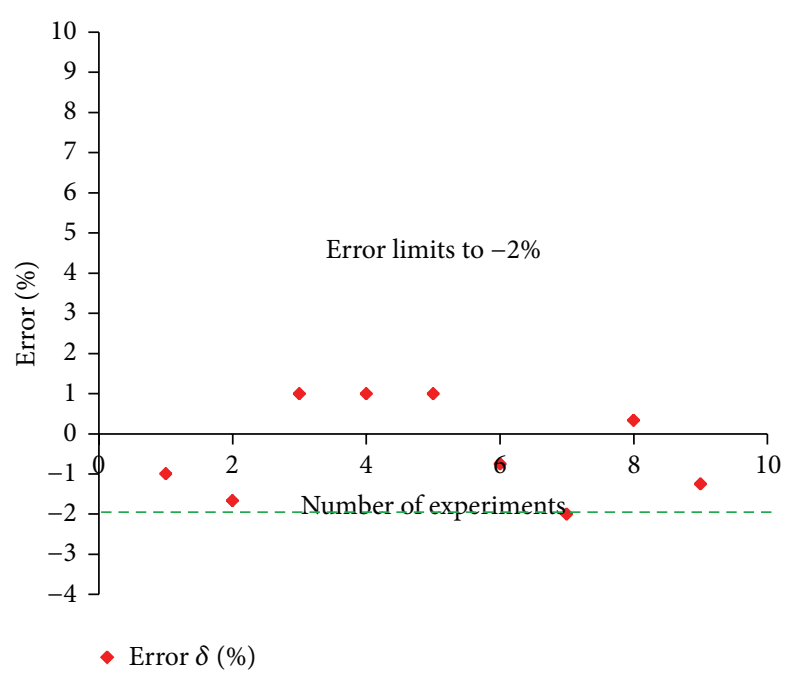

(b)

FIGURE 15: (a) Residual plot of crack location; (b) residual plot of crack depth in fixed-fixed beam.

Non-dimensional crack depth, $\delta$

$$
=\frac{\int(\text { depth }) \cdot \mu_{\mathrm{rcd}}(\text { depth }) \cdot d(\text { depth })}{\int \mu_{\mathrm{rcd}}(\text { depth }) \cdot d(\text { depth })} .
$$

In the above fuzzy analysis, the controller accepts the inputs and maps them into their membership functions and truth values. These mappings are then fed into the rules. If the rule specifies an AND relationship between the mappings of the six input variables, as shown in Figure 7, the minimum of the six is used as the combined truth value; if an OR is specified, the maximum is used. The appropriate output state is selected and assigned a membership value at the truth level of the premise. The truth values are then defuzzified and actual value is obtained.

\section{Results and Discussions}

In the proposed research work the results have been obtained from finite element analysis, experimental analysis, and fuzzy inference system for crack location and depth in a fixedfixed aluminum alloy beam structure. Based on the results obtained from all the aforementioned methodologies, the following discussions can be made. 
TABLE 2: Linguistic terms used for membership functions.

\begin{tabular}{|c|c|c|}
\hline $\begin{array}{l}\text { Name of the membership } \\
\text { function }\end{array}$ & Linguistic terms & Description and range of the linguistic terms \\
\hline L1F1, L1F2, L1F3, L1F4 & fnf $_{1 \text { to } 4}$ & $\begin{array}{l}\text { Low ranges of relative natural frequency for first mode of vibration in ascending } \\
\text { order, respectively }\end{array}$ \\
\hline M1F1, M1F2 & $\mathrm{fnf}_{5 \text { to } 6}$ & $\begin{array}{l}\text { Medium ranges of relative natural frequency for first mode of vibration in } \\
\text { ascending order, respectively }\end{array}$ \\
\hline $\mathrm{H} 1 \mathrm{~F} 1, \mathrm{H} 1 \mathrm{~F} 2, \mathrm{H} 1 \mathrm{~F} 3, \mathrm{H} 1 \mathrm{~F} 4$ & $\operatorname{fnf}_{7 \text { to } 10}$ & $\begin{array}{l}\text { Higher ranges of relative natural frequency for first mode of vibration in ascending } \\
\text { order, respectively }\end{array}$ \\
\hline L2F1, L2F2, L2F3, L2F4 & $\operatorname{snf}_{1 \text { to } 4}$ & $\begin{array}{l}\text { Low ranges of relative natural frequency for second mode of vibration in ascending } \\
\text { order, respectively }\end{array}$ \\
\hline $\mathrm{M} 2 \mathrm{~F} 1, \mathrm{M} 2 \mathrm{~F} 2$ & $\operatorname{snf}_{5 \text { to } 6}$ & $\begin{array}{l}\text { Medium ranges of relative natural frequency for second mode of vibration in } \\
\text { ascending order, respectively }\end{array}$ \\
\hline $\mathrm{H} 2 \mathrm{~F} 1, \mathrm{H} 2 \mathrm{~F} 2, \mathrm{H} 2 \mathrm{~F} 3, \mathrm{H} 2 \mathrm{~F} 4$ & $\operatorname{snf}_{7 \text { to } 10}$ & $\begin{array}{l}\text { Higher ranges of relative natural frequency for second mode of vibration in } \\
\text { ascending order, respectively }\end{array}$ \\
\hline L3F1, L3F2, L3F3, L3F4 & $\operatorname{tnf}_{1 \text { to } 4}$ & $\begin{array}{l}\text { Low ranges of relative natural frequency for third mode of vibration in ascending } \\
\text { order, respectively }\end{array}$ \\
\hline M3F1, M3F2 & $\operatorname{tnf}_{5}$ to 6 & $\begin{array}{l}\text { Medium ranges of relative natural frequency for third mode of vibration in } \\
\text { ascending order, respectively }\end{array}$ \\
\hline $\mathrm{H} 3 \mathrm{~F} 1, \mathrm{H} 3 \mathrm{~F} 2, \mathrm{H} 3 \mathrm{~F} 3, \mathrm{H} 3 \mathrm{~F} 4$ & $\operatorname{tnf}_{7 \text { to } 10}$ & $\begin{array}{l}\text { Higher ranges of relative natural frequency for third mode of vibration in ascending } \\
\text { order, respectively }\end{array}$ \\
\hline L1M1, L1M2, L1M3, L1M4 & $\mathrm{fms}_{1 \text { to } 4}$ & $\begin{array}{l}\text { Low ranges of mode shape deflection for first mode of vibration in ascending order, } \\
\text { respectively }\end{array}$ \\
\hline M1M1, M1M2 & $\mathrm{fms}_{5 \text { to } 6}$ & $\begin{array}{l}\text { Medium ranges of mode shape deflection for first mode of vibration in ascending } \\
\text { order, respectively }\end{array}$ \\
\hline $\begin{array}{l}\text { H1M1, H1M2, H1M3, } \\
\text { H1M4 }\end{array}$ & $\mathrm{fms}_{7}$ to 10 & $\begin{array}{l}\text { Higher ranges of mode shape deflection for first mode of vibration in ascending } \\
\text { order, respectively }\end{array}$ \\
\hline $\begin{array}{l}\text { L2M1, L2M2, L2M3, } \\
\text { L2M4 }\end{array}$ & $\mathrm{sms}_{1 \text { to } 4}$ & $\begin{array}{l}\text { Low ranges of mode shape deflection for second mode of vibration in ascending } \\
\text { order, respectively }\end{array}$ \\
\hline M2M1, M2M2 & $\mathrm{sms}_{5 \text { to } 6}$ & $\begin{array}{l}\text { Medium ranges of mode shape deflection for second mode of vibration in } \\
\text { ascending order, respectively }\end{array}$ \\
\hline $\begin{array}{l}\mathrm{H} 2 \mathrm{M} 1, \mathrm{H} 2 \mathrm{M} 2, \mathrm{H} 2 \mathrm{M} 3 \\
\mathrm{H} 2 \mathrm{M} 4\end{array}$ & $\mathrm{sms}_{7 \text { to } 10}$ & $\begin{array}{l}\text { Higher ranges of mode shape deflection for second mode of vibration in ascending } \\
\text { order, respectively }\end{array}$ \\
\hline $\begin{array}{l}\text { L3M1, L3M2, L3M3, } \\
\text { L3M4 }\end{array}$ & $\mathrm{tms}_{1 \text { to } 4}$ & $\begin{array}{l}\text { Low ranges of mode shape deflection for third mode of vibration in ascending } \\
\text { order, respectively }\end{array}$ \\
\hline M3M1, M3M2 & $\mathrm{tms}_{5}$ to 6 & $\begin{array}{l}\text { Medium ranges of mode shape deflection for third mode of vibration in ascending } \\
\text { order, respectively }\end{array}$ \\
\hline $\begin{array}{l}\text { H3M1, H3M2, H3M3, } \\
\text { H3M4 }\end{array}$ & $\mathrm{tms}_{7 \text { to } 10}$ & $\begin{array}{l}\text { Higher ranges of mode shape deflection for third mode of vibration in ascending } \\
\text { order, respectively }\end{array}$ \\
\hline SD1, SD2, SD3, SD4 & $\operatorname{rcd}_{1 \text { to } 4}$ & Small ranges of relative crack depth in ascending order, respectively \\
\hline MD1, MD2 & $\operatorname{rcd}_{5 \text { to } 6}$ & Medium ranges of relative crack depth in ascending order, respectively \\
\hline $\mathrm{HD} 1, \mathrm{HD} 2, \mathrm{HD} 3, \mathrm{HD} 4$ & $\operatorname{rcd}_{7 \text { to } 10}$ & Larger ranges of relative crack depth in ascending order, respectively \\
\hline SL1, SL2, SL3, SL4 & $\mathrm{rcl}_{1 \text { to } 4}$ & Small ranges of relative crack location in ascending order, respectively \\
\hline ML1, ML2 & $\mathrm{rcl}_{5 \text { to } 6}$ & Medium ranges of relative crack location in ascending order, respectively \\
\hline HL1, HL2, HL3, HL4 & $\mathrm{rcl}_{7 \text { to } 10}$ & Bigger ranges of relative crack location in ascending order \\
\hline
\end{tabular}

4.1. Numerical Results. The analysis of the cracked beam structure from which the frequency values are obtained has been performed in ANSYS 13.0 [24] by keeping the crack depth constant and varying the crack location and vice versa. Typical frequency response plots have been plotted as shown in Figures 8 and 9. Figure 8 shows the variation of relative natural frequency plotted against relative crack location $(\alpha)$ for four sets of crack depths $(\delta=0.2,0.3,0.4$, and 0.5$)$ for first, second, and third modes of vibration. The portion of the minimum frequency value depends upon the mode shape at $0.5 \mathrm{rcl}$ for 1st mode, at 0.25 and $0.75 \mathrm{rcl}$ for the $2 \mathrm{nd}$ mode, and at $0.17,0.5$, and $0.83 \mathrm{rcl}$ for the $3 \mathrm{rd}$ mode of vibration. The frequency of the uncracked and cracked beam matches for the $\mathrm{rcl}$ at the node point of the vibration modes irrespective 
TABLE 3: Some of the fuzzy rules used in the analysis.

\begin{tabular}{|c|c|}
\hline Sl. number & Fuzzy rules \\
\hline 1 & If $\mathrm{fnf}$ is $\mathrm{L} 1 \mathrm{~F} 1$, snf is $\mathrm{L} 2 \mathrm{~F} 1$, tnf is $\mathrm{L} 3 \mathrm{~F} 1, \mathrm{fms}$ is $\mathrm{L} 1 \mathrm{M} 2$, sms is $\mathrm{L} 2 \mathrm{M} 4$, and tms is HLM3, then $\mathrm{rcd}$ is SD2 and $\mathrm{rcl}$ is SL1. \\
\hline 2 & If $\mathrm{fnf}$ is $\mathrm{L} 1 \mathrm{~F} 4$, snf is $\mathrm{L} 2 \mathrm{~F} 3$, tnf is $\mathrm{L} 3 \mathrm{~F} 2, \mathrm{fms}$ is $\mathrm{H} 1 \mathrm{M} 3$, sms is M2M1, and tms is $\mathrm{H} 3 \mathrm{M} 4$, then rcd is SD3 and rcl is HL1. \\
\hline 3 & If $\mathrm{fnf}$ is $\mathrm{L} 1 \mathrm{~F} 3$, snf is $\mathrm{L} 2 \mathrm{~F} 4$, tnf is $\mathrm{L} 3 \mathrm{~F} 4, \mathrm{fms}$ is M1M2, sms is $\mathrm{H} 2 \mathrm{M} 2$, and tmd is H3M3, then rcd is MD1 and $\mathrm{rcl}$ is HL3. \\
\hline 4 & If $\mathrm{fnf}$ is $\mathrm{H} 1 \mathrm{~F} 2$, snf is $\mathrm{H} 2 \mathrm{~F} 1$, tnf is $\mathrm{H} 3 \mathrm{~F} 1$, fms is $\mathrm{H} 1 \mathrm{M} 3$, sms is $\mathrm{H} 2 \mathrm{M} 4$, and tms is $\mathrm{H} 3 \mathrm{M} 4$, then $\mathrm{rcd}$ is SD4 and rcl is SL11. \\
\hline 5 & If $\mathrm{fnf}$ is $\mathrm{M} 1 \mathrm{~F} 1$, snf is $\mathrm{L} 2 \mathrm{~F} 2$, $\mathrm{tnf}$ is $\mathrm{L} 3 \mathrm{~F} 3, \mathrm{fms}$ is $\mathrm{H} 1 \mathrm{M} 1$, sms is $\mathrm{H} 2 \mathrm{M} 1$, and tms is $\mathrm{H} 3 \mathrm{M} 2$, then $\mathrm{rcd}$ is SD4 and $\mathrm{rcl}$ is SL3. \\
\hline 6 & If $\mathrm{fnf}$ is $\mathrm{L} 1 \mathrm{~F} 1$, snf is $\mathrm{L} 2 \mathrm{~F} 2$, tnf is $\mathrm{L} 3 \mathrm{~F} 3$, fms is $\mathrm{H} 1 \mathrm{M} 3$, sms is M2M1, and tms is $\mathrm{H} 3 \mathrm{M} 4$, then $\mathrm{rcd}$ is MD1 and $\mathrm{rcl}$ is SL4. \\
\hline 7 & If $\mathrm{fnf}$ is $\mathrm{L} 1 \mathrm{~F} 4$, snf is $\mathrm{L} 2 \mathrm{~F} 4$, tnf is $\mathrm{L} 3 \mathrm{~F} 4, \mathrm{fms}$ is $\mathrm{M} 1 \mathrm{M} 2$, sms is $\mathrm{H} 2 \mathrm{M} 1$, and tms is $\mathrm{H} 3 \mathrm{M} 1$, then $\mathrm{rcd}$ is $\mathrm{HD} 1$ and $\mathrm{rcl}$ is ML1. \\
\hline 8 & If $\mathrm{fnf}$ is $\mathrm{H} 1 \mathrm{~F} 1$, snf is $\mathrm{M} 2 \mathrm{~F} 2$, $\mathrm{tnf}$ is $\mathrm{M} 3 \mathrm{~F} 1$, $\mathrm{fms}$ is $\mathrm{H} 1 \mathrm{M} 2$, sms is $\mathrm{H} 2 \mathrm{M} 2$, and tms is $\mathrm{H} 3 \mathrm{M} 2$, then $\mathrm{rcd}$ is MD2 and rcl is HL1. \\
\hline 9 & If $\mathrm{fnf}$ is $\mathrm{L} 1 \mathrm{~F} 1, \mathrm{snf}$ is $\mathrm{L} 2 \mathrm{~F} 4, \mathrm{tnf}$ is $\mathrm{L} 3 \mathrm{~F} 4, \mathrm{fms}$ is M1M1, sms is M2M1, and tms is M3M2, then $\mathrm{rcd}$ is SD2 and rcl is SL4. \\
\hline 10 & If $\mathrm{fnf}$ is $\mathrm{M} 1 \mathrm{~F} 1$, snf is $\mathrm{L} 2 \mathrm{~F} 2$, $\mathrm{tnf}$ is $\mathrm{L} 3 \mathrm{~F} 1, \mathrm{fms}$ is M1M2, sms is M2M2, and tms is H3M1, then $\mathrm{rcd}$ is SD3 and $\mathrm{rcl}$ is HL2. \\
\hline
\end{tabular}

TABLE 4: Computed nondimensional natural frequencies and mode shape differences of fixed-fixed beam.

\begin{tabular}{lcccccccc}
\hline \multirow{2}{*}{ Sl. number } & \multicolumn{2}{c}{ Test points } & \multicolumn{3}{c}{ Nondimensional natural frequency } & \multicolumn{3}{c}{ Nondimensional mode shape differences } \\
& $\alpha$ & $\delta$ & $\bar{\Delta}_{\mathrm{nf}_{1}}$ & $\bar{\Delta}_{\mathrm{nf}_{2}}$ & $\bar{\Delta}_{\mathrm{nf}_{3}}$ & $\bar{\Delta}_{m_{1}}$ & $\bar{\Delta}_{m_{2}}$ & $\bar{\Delta}_{m_{3}}$ \\
\hline 1 & Uncrack & 0.000 & 1.000 & 1.000 & 1.000 & 0.000 & 0.000 & 0.000 \\
2 & 0.125 & 0.200 & 0.939 & 0.954 & 0.941 & 0.265 & 0.239 & 0.302 \\
3 & 0.125 & 0.300 & 0.925 & 0.951 & 0.939 & 0.373 & 0.305 & 0.373 \\
4 & 0.125 & 0.400 & 0.912 & 0.943 & 0.949 & 0.162 & 0.025 & 0.081 \\
5 & 0.250 & 0.200 & 0.933 & 0.956 & 0.942 & 0.127 & -0.085 & 0.174 \\
6 & 0.250 & 0.300 & 0.926 & 0.949 & 0.941 & 0.017 & 0.811 & 0.234 \\
7 & 0.250 & 0.400 & 0.919 & 0.949 & 0.927 & 0.182 & 0.057 & 0.404 \\
9 & 0.375 & 0.200 & 0.926 & 0.941 & 0.945 & 0.075 & 0.224 & 0.372 \\
10 & 0.375 & 0.300 & 0.921 & 0.941 & 0.939 & 0.077 & 0.309 & 0.379 \\
\hline
\end{tabular}

TABLE 5: Measured nondimensional natural frequencies and mode shape differences of fixed-fixed beam.

\begin{tabular}{lcccccccc}
\hline \multirow{2}{*}{ Sl. number } & \multicolumn{2}{c}{ Test points } & \multicolumn{3}{c}{ Nondimensional natural frequency } & \multicolumn{3}{c}{ Nondimensional mode shape differences } \\
& $\alpha$ & $\delta$ & $\bar{\Delta}_{\mathrm{nf}}$ & $\bar{\Delta}_{\mathrm{nf}}$ & $\bar{\Delta}_{\mathrm{nf}}$ & $\bar{\Delta}_{m_{1}}$ & $\bar{\Delta}_{m_{2}}$ & $\bar{\Delta}_{m_{3}}$ \\
\hline 1 & Uncrack & 0.000 & 1.000 & 1.000 & 1.000 & 0.000 & 0.000 & 0.000 \\
2 & 0.125 & 0.200 & 0.935 & 0.957 & 0.952 & 0.262 & 0.226 & 0.309 \\
3 & 0.125 & 0.300 & 0.924 & 0.955 & 0.95 & 0.378 & 0.315 & 0.378 \\
4 & 0.125 & 0.400 & 0.909 & 0.943 & 0.948 & 0.175 & 0.034 & 0.082 \\
5 & 0.250 & 0.200 & 0.941 & 0.962 & 0.955 & 0.141 & -0.082 & 0.189 \\
6 & 0.250 & 0.300 & 0.919 & 0.961 & 0.939 & 0.012 & -0.805 & 0.242 \\
7 & 0.250 & 0.400 & 0.932 & 0.957 & 0.937 & 0.178 & 0.059 & 0.372 \\
9 & 0.375 & 0.200 & 0.937 & 0.95 & 0.954 & 0.065 & 0.239 & 0.394 \\
10 & 0.375 & 0.300 & 0.929 & 0.948 & 0.951 & 0.078 & 0.291 & 0.376 \\
\hline
\end{tabular}


TABLE 6: Comparison of results among the various membership functions.

\begin{tabular}{lcccccccccc}
\hline \multirow{2}{*}{ Sl. number } & \multicolumn{2}{c}{ Triangular } & \multicolumn{2}{c}{ Trapezoidal } & \multicolumn{2}{c}{ Gaussian } & \multicolumn{3}{c}{ Hybrid } & \multicolumn{2}{c}{ Experiment } \\
& $\delta$ & $\alpha$ & $\delta$ & $\alpha$ & $\delta$ & $\alpha$ & $\delta$ & $\alpha$ & $\delta$ \\
\hline 1 & 0.121 & 0.189 & 0.120 & 0.192 & 0.120 & 0.190 & 0.123 & 0.202 & 0.125 & 0.200 \\
2 & 0.129 & 0.289 & 0.128 & 0.280 & 0.131 & 0.282 & 0.127 & 0.305 & 0.125 & 0.300 \\
3 & 0.132 & 0.391 & 0.131 & 0.392 & 0.119 & 0.389 & 0.122 & 0.396 & 0.125 & 0.400 \\
4 & 0.261 & 0.190 & 0.259 & 0.191 & 0.257 & 0.192 & 0.248 & 0.198 & 0.250 & 0.200 \\
5 & 0.258 & 0.310 & 0.261 & 0.308 & 0.241 & 0.309 & 0.246 & 0.297 & 0.250 & 0.300 \\
6 & 0.261 & 0.415 & 0.255 & 0.408 & 0.240 & 0.412 & 0.252 & 0.403 & 0.250 & 0.400 \\
7 & 0.372 & 0.212 & 0.386 & 0.207 & 0.381 & 0.209 & 0.371 & 0.204 & 0.375 & 0.200 \\
8 & 0.368 & 0.309 & 0.369 & 0.312 & 0.367 & 0.307 & 0.374 & 0.299 & 0.375 & 0.300 \\
9 & 0.387 & 0.415 & 0.381 & 0.406 & 0.383 & 0.409 & 0.379 & 0.405 & 0.375 & 0.400 \\
\hline
\end{tabular}

of crack depth. The comparison of relative natural frequency with respect to relative crack location for three modes of vibration for $\delta=0.2$ has been presented in Figure 8(d).

Figure 9 shows the variation of relative natural frequencies versus relative crack depth for four sets of crack locations $(\alpha=0.2,0.4,0.6$, and 0.8$)$. It can be seen that the frequency value decreases with the increase in crack depth. However, the effect of crack depth is marginal for the crack position close to the support ( $\alpha=0.2$ and 0.8 ) and the effect is more prominent for the crack located near middle span ( $\alpha=0.4$ and 0.6 ) beam. This variation is symmetric due to the symmetry in clamping. The variation of $\alpha=0.2$ matches $\alpha=0.8$ and $\alpha=0.4$ matches $\alpha=0.6$. Table 4 gives the variation of relative natural frequencies of a fixed-fixed beam for different crack location and crack depth.

4.2. Experimental Results. Experimental results have been used to show the mode shapes for uncracked and cracked beam as shown in Figure 10. The variation of amplitudes with respect to the crack location has also been shown in this figure. Figures 10(a), 10(b), and 10(c) show the mode shapes for uncracked and cracked beam condition for 1st, 2nd, and 3 rd modes of vibration, respectively. The variation in mode shapes is more prominent in higher mode of vibration. It has been observed from the figures that the deviations in mode shape are more for a higher depth of crack. In Figure 10 the mode-shape variation has been shown at the relative crack location of 0.125 and the respective relative crack depths 0.167 and 0.333 for crack and crack 2 .

Figure 11 shows the three mode shapes of a fixed-fixed beam with a relative crack depth of 0.167 at the relative crack location 0.25 . It shows that the mode-shape variation is more with the change of relative crack location towards center of the beam by keeping relative crack depth constant. The results obtained from the experimental analysis have been presented in Table 5.

4.3. Simulated Results. In the third phase of the proposed research work, fuzzy analysis has been carried out using MATLAB [27]. By analysing the results, various surface plots have been obtained as shown in Figures 12 and 13. The smoothness of the surface plot indicates the variation of output with inputs. It is concluded from the surface plot that the contribution of interdependence parameters toward obtaining the output can easily provided through fuzzy inference system. The different surface plots have been obtained for the relative crack depth and relative crack location by varying the 2 nd natural frequency and $3 \mathrm{rd}$ natural frequency and the relative amplitudes with the first natural frequency.

To validate the use of hybrid membership function in the proposed work a comparison has been made between the results obtained from triangular, trapezoidal, and Gaussian membership function with hybrid membership function and the result has been shown in Table 6. It can be seen that results of the hybrid membership function are more close to experimental results.

In the third phase of the proposed work, experimental analysis has been carried out to test the robustness of the fuzzy inference system. Comparisons of the results obtained from the various analyses have been depicted in Table 7. The results of the developed fuzzy controller have been found to be in excellent matching with numerical and experimental values.

Figure 14 represents the comparison of results for relative crack depth and relative crack location for the three sets of analysis (fuzzy controller, FEM, and experimental) as mentioned in Table 7. It has been observed that the fuzzy controller gives results that are very close to those obtained using experimental analysis.

In the last phase of the proposed work, error data have been generated in comparison with fuzzy system and experimental analysis. To lay out the error and to check the robustness of the method, residual plots have been plotted as shown in Figure 15. The error deviation of the results mentioned has been shown in Table 8 . It can be seen that the error is within $2.4 \%$ for $\alpha$ and $-2 \%$ for $\delta$. Less error in prediction of crack location $(\alpha)$ has been observed in comparison with error in crack depth $(\delta)$. It is because of the fact that the variation of depth $(6 \mathrm{~mm})$ is very small in comparison with length $(800 \mathrm{~mm})$ as shown in Table 1.

Residuals are the difference between the predicted output from the model and the measured output from the validation data set. The residual plot is a graph that shows the residuals 
TABLE 7: Comparison of results.

\begin{tabular}{lcccccccccc}
\hline \multirow{2}{*}{ Sl. number } & \multicolumn{2}{c}{ Nondimensional Natural Frequency } & \multicolumn{3}{c}{ Nondimensional mode shape difference } & \multicolumn{3}{c}{ Hybrid fuzzy controller } & \multicolumn{3}{c}{ Experimental } \\
& $\bar{\Delta}_{\mathrm{nf}_{1}}$ & $\bar{\Delta}_{\mathrm{nf} 2}$ & $\bar{\Delta}_{\mathrm{nf}}$ & $\bar{\Delta}_{m_{1}}$ & $\bar{\Delta}_{m_{2}}$ & $\bar{\Delta}_{m_{3}}$ & $\alpha$ & & $\delta$ & $\alpha$ \\
\hline 1 & 0.935 & 0.957 & 0.952 & 0.262 & 0.226 & 0.309 & 0.123 & 0.202 & 0.125 & 0.200 \\
2 & 0.924 & 0.955 & 0.95 & 0.378 & 0.315 & 0.378 & 0.127 & 0.305 & 0.125 & 0.300 \\
3 & 0.909 & 0.943 & 0.948 & 0.175 & 0.034 & 0.082 & 0.122 & 0.396 & 0.125 & 0.400 \\
4 & 0.941 & 0.962 & 0.955 & 0.141 & -0.082 & 0.189 & 0.248 & 0.198 & 0.250 & 0.200 \\
5 & 0.919 & 0.961 & 0.939 & 0.012 & -0.805 & 0.242 & 0.246 & 0.297 & 0.250 & 0.300 \\
6 & 0.932 & 0.957 & 0.937 & 0.178 & 0.059 & 0.372 & 0.252 & 0.403 & 0.25 & 0.400 \\
7 & 0.937 & 0.95 & 0.954 & 0.065 & 0.239 & 0.394 & 0.371 & 0.204 & 0.375 & 0.200 \\
8 & 0.929 & 0.948 & 0.951 & 0.078 & 0.291 & 0.376 & 0.374 & 0.299 & 0.375 & 0.300 \\
9 & 0.931 & 0.942 & 0.949 & -0.035 & 0.296 & 0.390 & 0.379 & 0.405 & 0.375 & 0.400 \\
\hline
\end{tabular}

TABLE 8: Error deviation table.

\begin{tabular}{|c|c|c|c|c|c|c|}
\hline \multirow{2}{*}{ Sl. number } & \multicolumn{2}{|c|}{ Data obtained From experimental analysis } & \multicolumn{2}{|c|}{ Data obtained from hybrid Fuzzy Logic technique } & \multicolumn{2}{|c|}{$\%$ error deviation } \\
\hline & $\alpha$ & $\delta$ & $\alpha$ & $\delta$ & $\alpha$ & $\delta$ \\
\hline 1 & 0.125 & 0.200 & 0.123 & 0.202 & 1.600 & -1.000 \\
\hline 2 & 0.125 & 0.300 & 0.127 & 0.305 & -1.600 & -1.667 \\
\hline 3 & 0.125 & 0.400 & 0.122 & 0.396 & 2.400 & 1.000 \\
\hline 4 & 0.25 & 0.200 & 0.248 & 0.198 & 0.800 & 1.000 \\
\hline 5 & 0.25 & 0.300 & 0.246 & 0.297 & 1.600 & 1.000 \\
\hline 6 & 0.25 & 0.400 & 0.252 & 0.403 & -0.800 & -0.750 \\
\hline 7 & 0.375 & 0.200 & 0.371 & 0.204 & 1.067 & -2.000 \\
\hline 8 & 0.375 & 0.300 & 0.374 & 0.299 & 0.267 & 0.333 \\
\hline 9 & 0.375 & 0.400 & 0.379 & 0.405 & -1.067 & -1.250 \\
\hline
\end{tabular}

in the vertical axis and the number of experiments in the horizontal axis. The residual plots of testing data of all the outputs are obtained and it has been concluded that the data obtained from fuzzy inference system have reasonably low error. Figures 15(a) and 15(b) show the residual plot for outputs $\delta$ and $\alpha$.

\section{Conclusion}

In this paper a new hybrid fuzzy inference system for crack detection of fixed-fixed beam using numerical and experimental data is presented. It is observed that the contribution of interdependence parameters toward obtaining the output can easily be provided through fuzzy inference system. The proposed hybrid membership function was found to be more efficient compared to triangular, trapezoidal, and Gaussian membership function. As per the experimental database the error is within $2.4 \%$ for the nondimensional crack location, $\alpha$, and $-2 \%$ for the nondimensional crack depth, $\delta$. Hence, the developed hybrid membership fuzzy controller is recommended to be used for the identification of cracks in continuous system. The present study for investigation of crack in a beam structure may be a source of inspiration for future work in different structures with various end conditions.

\section{Conflict of Interests}

The authors declare that there is no conflict of interests regarding the publication of this paper.

\section{References}

[1] M.-B. Shim and M.-W. Suh, "Crack identification of a planar frame structure based on a synthetic artificial intelligence technique," International Journal for Numerical Methods in Engineering, vol. 57, no. 1, pp. 57-82, 2003.

[2] R. Ganguli, "A fuzzy logic system for ground based structural health monitoring of a helicopter rotor using modal data," Journal of Intelligent Material Systems and Structures, vol. 12, no. 6, pp. 397-407, 2001.

[3] H. Gu and G. Song, "Active vibration suppression of a composite I-beam using fuzzy positive position control," Smart Materials and Structures, vol. 14, no. 4, pp. 540-547, 2005.

[4] P. K. Jena, D. N. Thatoi, and D. R. Parhi, "Differential evolution: an inverse approach for crack detection," Advances in Acoustics and Vibration, vol. 2013, Article ID 321931, 10 pages, 2013.

[5] J. Lin and W.-Z. Liu, "Experimental evaluation of a piezoelectric vibration absorber using a simplified fuzzy controller in a cantilever beam," Journal of Sound and Vibration, vol. 296, no. 3, pp. 567-582, 2006. 
[6] M. Chandrashekhar and R. Ganguli, "Damage assessment of structures with uncertainty by using mode-shape curvatures and fuzzy logic," Journal of Sound and Vibration, vol. 326, no. 35, pp. 939-957, 2009.

[7] Y. M. Kim, C. K. Kim, and G. H. Hong, "Fuzzy set based crack diagnosis system for reinforced concrete structures," Computers and Structures, vol. 85, no. 23-24, pp. 1828-1844, 2007.

[8] D. Thatoi, P. Guru, P. K. Jena, S. Choudhury, and H. C. Das, "Comparison of CFBP, FFBP, and RBF networks in the field of crack detection," Modelling and Simulation in Engineering, vol. 2014, Article ID 292175, 13 pages, 2014.

[9] N. Saravanan, V. Siddabattuni, and K. I. Ramachandran, "Fault diagnosis of spur bevel gear box using artificial neural network (ANN), and proximal support vector machine (PSVM)," Applied Soft Computing Journal, vol. 10, no. 1, pp. 344-360, 2010.

[10] T. Boutros and M. Liang, "Mechanical fault detection using fuzzy index fusion," International Journal of Machine Tools and Manufacture, vol. 47, no. 11, pp. 1702-1714, 2007.

[11] Q. Wu and R. Law, "Complex system fault diagnosis based on a fuzzy robust wavelet support vector classifier and an adaptive Gaussian particle swarm optimization," Information Sciences, vol. 180, no. 23, pp. 4514-4528, 2010.

[12] V. Sugumaran and K. I. Ramachandran, "Fault diagnosis of roller bearing using fuzzy classifier and histogram features with focus on automatic rule learning," Expert Systems with Applications, vol. 38, no. 5, pp. 4901-4907, 2011.

[13] L. J. De Miguel and L. F. Blázquez, "Fuzzy logic-based decisionmaking for fault diagnosis in a DC motor," Engineering Applications of Artificial Intelligence, vol. 18, no. 4, pp. 423-450, 2005.

[14] S. D. Nguyen, K. N. Ngo, Q. T. Tran, and S.-B. Choi, "A new method for beam-damage-diagnosis using adaptive fuzzy neural structure and wavelet analysis," Mechanical Systems and Signal Processing, vol. 39, no. 1-2, pp. 181-194, 2013.

[15] Y. Serhat Erdogan and P. Gundes Bakir, "Inverse propagation of uncertainties in finite element model updating through use of fuzzy arithmetic," Engineering Applications of Artificial Intelligence, vol. 26, no. 1, pp. 357-367, 2013.

[16] D. N. Thatoi, H. C. Das, and D. R. Parhi, "Review of techniques for fault diagnosis in damaged structure and engineering system," Advances in Mechanical Engineering, vol. 2012, Article ID 327569, 11 pages, 2012.

[17] P. Beena and R. Ganguli, "Structural damage detection using fuzzy cognitive maps and Hebbian learning," Applied Soft Computing Journal, vol. 11, no. 1, pp. 1014-1020, 2011.

[18] K. V. Reddy and R. Ganguli, "Fourier analysis of mode shapes of damaged beams," Computers, Materials and Continua, vol. 5, no. 2, pp. 79-97, 2007.

[19] S.-J. Zheng, Z.-Q. Li, and H.-T. Wang, "A genetic fuzzy radial basis function neural network for structural health monitoring of composite laminated beams," Expert Systems with Applications, vol. 38, no. 9, pp. 11837-11842, 2011.

[20] J. P. Sawyer and S. S. Rao, "Structural damage detection and identification using fuzzy logic," AIAA Journal, vol. 38 , no. 12, pp. 2328-2335, 2000.

[21] P. M. Pawar and R. Ganguli, "Genetic fuzzy system for damage detection in beams and helicopter rotor blades," Computer Methods in Applied Mechanics and Engineering, vol. 192, no. 1618, pp. 2031-2057, 2003.

[22] S. da Silva, M. Dias Júnior, V. Lopes Junior, and M. J. Brennan, "Structural damage detection by fuzzy clustering," Mechanical Systems and Signal Processing, vol. 22, no. 7, pp. 1636-1649, 2008.
[23] P. M. Pawar and R. Ganguli, Structural Health Monitoring Using Genetic Fuzzy Systems, Springer, London, UK, 2011.

[24] ANSYS, Academic Research, Release 13.0.

[25] D. R. Parhi, "Navigation of mobile robots using a fuzzy logic controller," Journal of Intelligent and Robotic Systems: Theory and Applications, vol. 42, no. 3, pp. 253-273, 2005.

[26] S. Sumathi and P. Surekha, Computational Intelligence Paradigms: Theory and Applications Using Matlab, CRC Press, Taylor \& Francis, 2010.

[27] The Mathworks, MATLAB. 7.0.1.24704. 

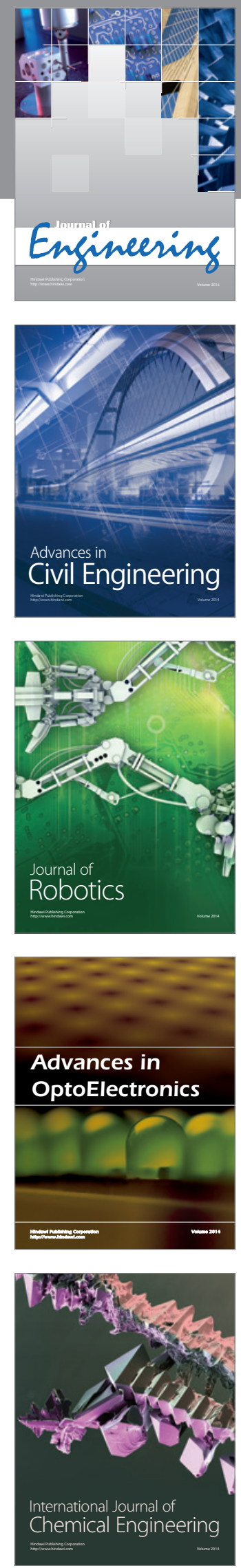

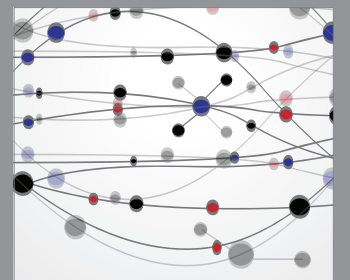

The Scientific World Journal
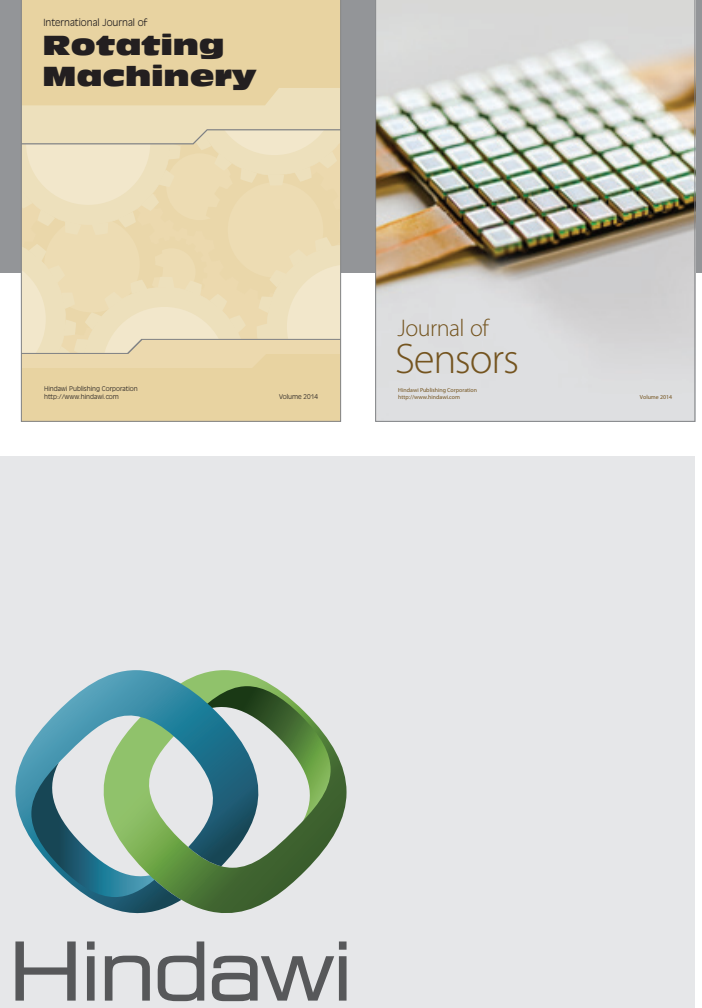

Submit your manuscripts at http://www.hindawi.com
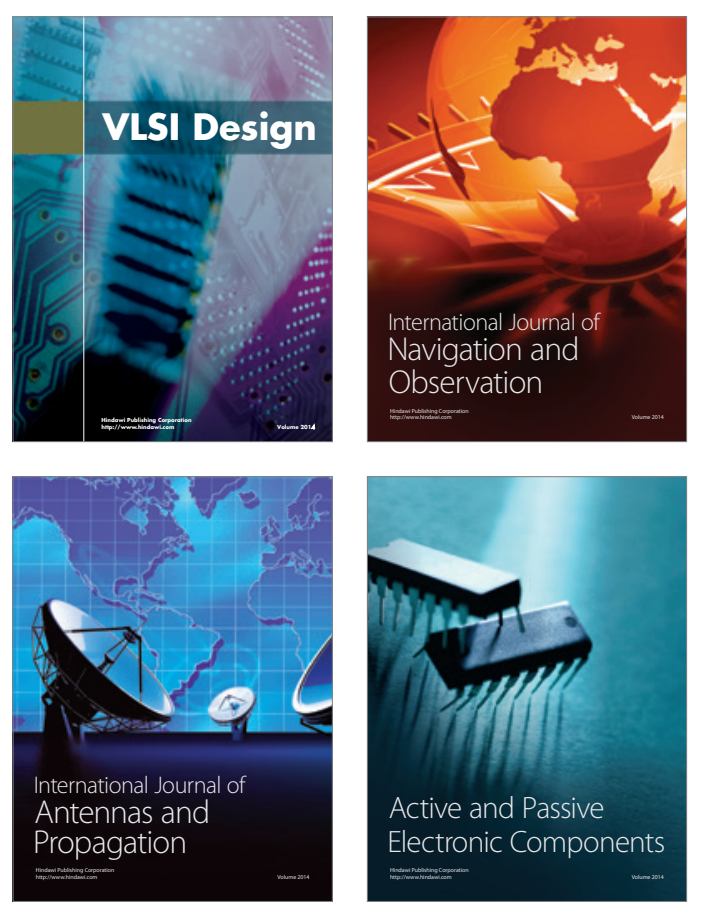
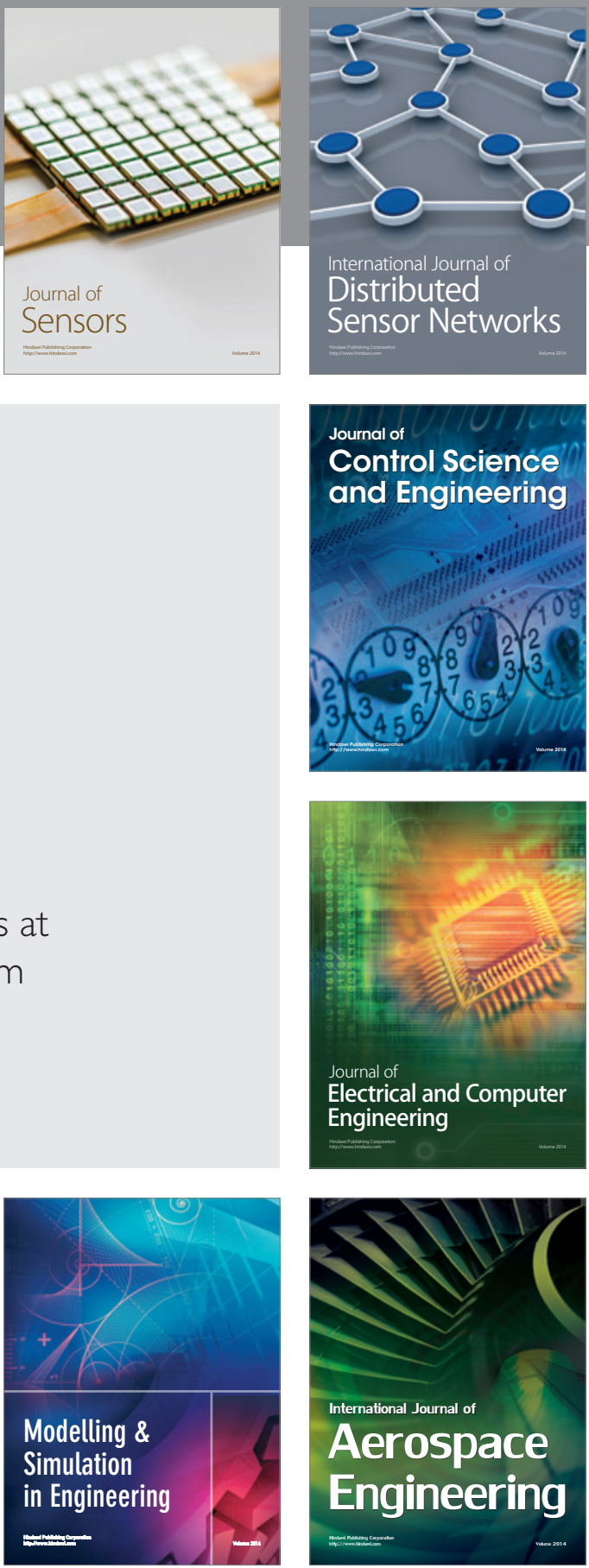

Journal of

Control Science

and Engineering
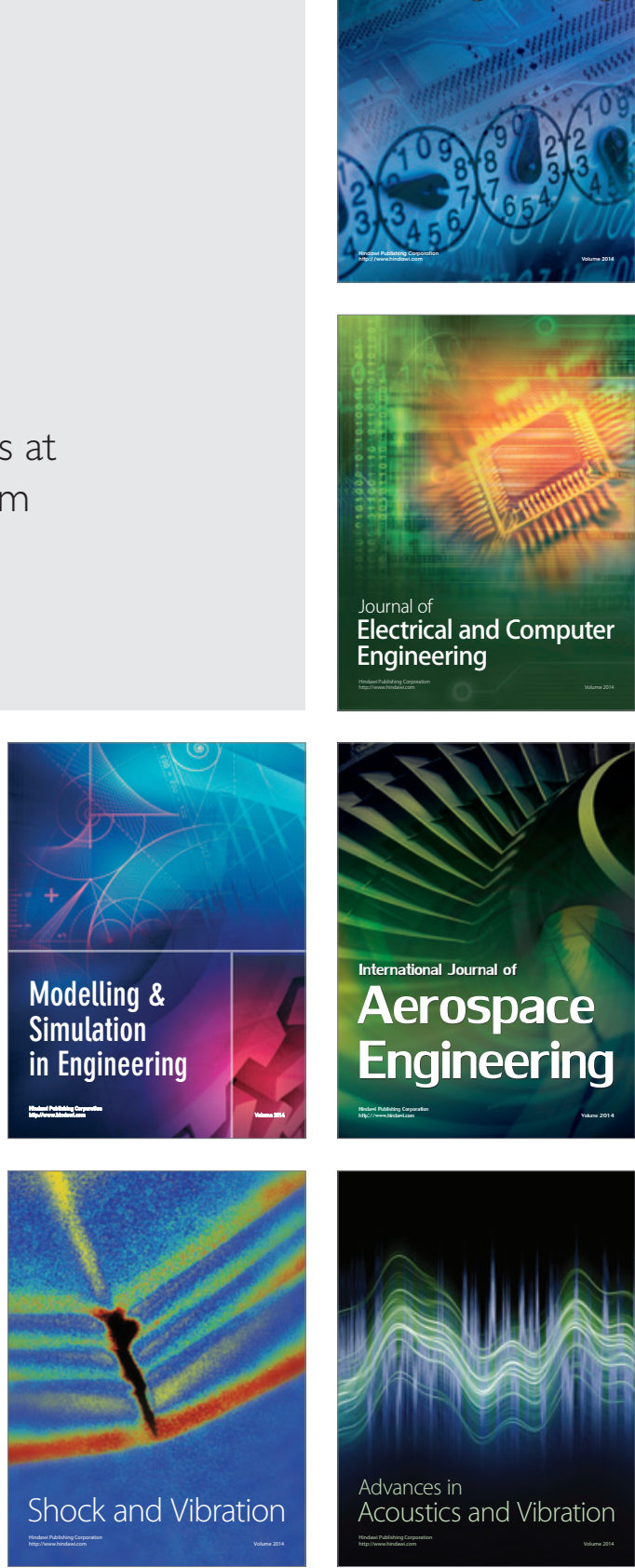